ÉGYPTE

monde arabe

\section{Égypte/Monde arabe}

8 | 1991

Perceptions de la centralité de l'Égypte 2

\title{
L'Égypte dans la politologie occidentale
}

\author{
Jean-Claude Vatin
}

\section{(2) OpenEdition}

Journals

Édition électronique

URL : https://journals.openedition.org/ema/1200

DOI : 10.4000/ema.1200

ISSN : 2090-7273

\section{Éditeur}

CEDEJ - Centre d'études et de documentation économiques juridiques et sociales

\section{Édition imprimée}

Date de publication : 31 décembre 1991

Pagination : 7-48

ISSN : 1110-5097

\section{Référence électronique}

Jean-Claude Vatin, «L'Égypte dans la politologie occidentale », Égypte/Monde arabe [En ligne], 8| 1991,

mis en ligne le 08 juillet 2008, consulté le 07 juillet 2022. URL : http://journals.openedition.org/ema/

1200 ; DOI : https://doi.org/10.4000/ema.1200

\section{Ce document a été généré automatiquement le 7 juillet 2022}

Tous droits réservés 


\title{
L'Égypte dans la politologie occidentale
}

\author{
Jean-Claude Vatin
}

\section{NOTE DE L'ÉDITEUR}

Communication au séminaire de recherche CEDEJ-IFAO, du 11 juin 1991. Version partielle d'une étude plus large, revue en fonction des remarques formulées alors, notamment par MM. Hassan Hanafi et Ahmed Abdallah.

1 Le thème retenu - pour s'inscrire dans le droit fil de la «centralité de l'Égypte » qui a servi à la fois d'emblème et de raison aux quelques trente séances du séminaire 1990-1991 organisé conjointement par le CEDEJ et l'IFAO - mérite d'être élucidé en quelques mots. Je me propose d'aborder, par divers biais, les modes de construction du savoir occidental sur l'Égypte du point de vue de la science politique. Il s'agit d'établir le rapport entre une science et un objet, une discipline spécifique et une société circonscrite spatialement (composant un État national, avec une population, un territoire, des frontières, des modes de fonctionnement, une organisation politique). Avec ceci de particulier que la science est: a) jeune, fragile, voire sans autonomie véritable et que, en regard, l'objet est ancien, solide, possède une masse spécifique, et b) étrangère à l'objet dont elle prétend traiter.

2 D'où, d'entrée, des distorsions et inadéquations, une série de « résistances » de ce qui est étudié par rapport au savoir qui entend l'appréhender.

3 Ceci dit, l'objectif principal n'est pas de juger de l'apport de la science politique occidentale à la connaissance de l'Égypte; il ne saurait être question de porter une appréciation sur des contenus, moins encore d'estimer le bien fondé et la qualité des études effectuées. Mais de saisir la part et place de l'Égypte dans la science politique occidentale, disons depuis la seconde guerre mondiale. En nous rapprochant du temps présent, c'est-à-dire jusqu'à la fin des années 1980 , ce qui correspond à la littérature disponible. 
Contrairement aux règles en la matière, je ne partirai pas d'une proposition interprétative ou d'une hypothèse dont je vérifierais la validité par la suite. Je vais plutôt recourir à une approche éclatée, sans caractère descriptif systématique. Et en changeant d'angle, chaque fois.

5 Le projet s'inscrit dans une entreprise plus vaste consistant à rassembler en faisceau les éléments glanés par le biais de cinq « observations » successives, permettant de :

61 - parler du centre de la politologie occidentale elle-même, de suivre son évolution, et de voir comment l'Égypte y a été insérée, en choisissant comme exemple le cas français ;

72 - changer la vision et, tout en conservant un point de vue extérieur, de centrer sur l'Égypte telle que la sociologie politique nord-américaine cette fois l'a abordée, l'a située par rapport au reste du monde arabe, l'a traitée aussi par le biais de l'enseignement: enseignement nord-américain aux USA mêmes mais aussi en Égypte par le canal de l'université américaine du Caire (AUC);

83 - confronter les manières et procédés par lesquels les États-Unis ont abordé l'Égypte en fonction du monde arabe et dont les Français ont étudié l'Algérie par rapport au Maghreb ;

94 - rapprocher deux disciplines et enseignements, l'anthropologie et la sociologie politique, et comparer comment deux disciplines ont, depuis un demi-siècle environ, saisi l'entité Égypte, quelle part elles lui ont respectivement attribuée, quelles fonctions elles lui ont fait remplir, quelle place elles lui ont réservée dans leurs démonstrations, dans l'alimentation des théories, l'enrichissement méthodologique...

105 - pouvoir explorer du côté de la production britannique, pour laquelle l'Égypte reste une référence de taille et lancer des coups de sonde en direction des études de science politique en Europe : Allemagne, Espagne, Italie, par exemple.

11 Ces approches croisées, par leur combinaison, devraient aider à la saisie de ce que nous cherchons à révéler. Dans le présent texte, je n'aborderai que les deux premières, soit d'abord l'Égypte dans la science politique occidentale à partir de l'exemple français et, ensuite, l'Égypte dans la sociologie politique nord-américaine ${ }^{1}$.

\section{L'exemple français : une étude à la marge}

En France la science politique a une tradition mais elle n'a pas d'ancêtre éponyme. Aux étudiants de la génération issue de la seconde guerre mondiale, divers maîtres ont proposé comme pères fondateurs des étrangers ; ce pouvait être Aristote, Ibn Khaldun, Machiavel, Karl Marx, Max Weber, parmi d'autres. Ceux de la génération suivante ont même cru que le créateur de la politologie, en tant que science, corps de connaissances construit à partir d'hypothèses pouvant être vérifiées, était un Américain contemporain, David Easton. La filière française n'était pas plus sûre, avec Jean Bodin ou Montesquieu, tantôt Condorcet, tantôt Tocqueville, Auguste Comte ou Durkheim et, plus près de nous, Carré de Malberg et André Siegfried. Et Maurice Duverger, il y a trente ans de cela, était assez persuadé que l'héritier de ce multilignage c'était luimême, et non Georges Burdeau, auteur d'un énorme traité en sept volumes qui restera le seul de son espèce jusqu'à ce que Madeleine Grawitz et Jean Leca nous en donnent (en 1985) un nouveau en quatre volumes, correspondant aux grands domaines, courants dominants et systèmes d'interprétation de notre époque. À la Fondation 
nationale des Sciences politiques, le sentiment prévaut encore aujourd'hui que la naissance de l'École libre des sciences politiques, en 1871, marque la véritable naissance de la discipline. Ce que disputent les enseignants des Facultés de Droit qui délivrent des doctorats de sciences politiques et économiques depuis 1895 .

Disons, pour faire bref, que les institutions commencent à se mettre en place à la fin du XIX $^{e}$ siècle (le premier congrès des sciences politiques date de 1900 , très précisément). Mais le politique, en tant que domaine autonomisable, requérant des théories, des méthodes et techniques d'analyse propres à un savoir positif, rationalisé, en tant que catégorie spécifique de l'univers social, c'est-à-dire différent de l'économique, du philosophique, du psychologique, du moral, de l'administratif, par exemple, le politique comme lieu d'une discipline nouvelle ne s'est affirmé vraiment qu'après la seconde guerre mondiale. C'est le moment où l'Ecole libre des sciences politiques est nationalisée et où émergent une Fondation (pour la recherche) et un Institut (pour l'enseignement), qui fourniront les instruments d'expression de la communauté scientifique, notamment La Revue française de Science politique.

La force principale de la Fondation est alors et demeure encore partiellement aujourd'hui, la sociologie électorale. Une sociologie très franco-française - dans la tradition du Tableau politique de la France de l'ouest d'André Siegfried (1913) - parce qu'appliquée aux comportements politiques depuis la révolution, aux traitements des résultats électoraux jusqu'aux années 1970 en tout cas et, depuis, aux sondages. Les études électorales restent un des fleurons de la FNSP. Avec une fascination, un temps, pour les partis politiques, avec comme point culminant l'ouvrage de Maurice Duverger, Les partis politiques (1951) et le contre-livre de Georges Lavau, Partis politiques et réalités sociales (1953).

15 Les institutions politiques qui ont débordé peu à peu les analyses proprement juridiques des grands textes constitutionnels et législatifs, l'histoire des idées politiques, l'étude des relations internationales, ont constitué alors autant de "tranches ", quasiment de sous-disciplines, en compétition plus ou moins ouvertes les unes avec les autres. Mais sans que les politologues soient jamais parvenus à s'assurer la maitrise du débat intellectuel et moins encore le monopole de l'explication de ce qui est d'essence politique. On l'a vu encore récemment, à propos de la guerre du Golfe, comme des émeutes d'Algérie auparavant, ce n'est pas aux spécialistes que les médias s'adressent pour éclairer l'opinion. Pour commenter les sondages ou les derniers résultats électoraux, ils régnent sans partage. Ils sont totalement légitimes, et on n'imagine pas une chaîne de télévision en France, au soir du premier ou du second tour, sans son chercheur-démonstrateur du Centre d'études de la vie politique française ! En sociologie des organisations, en matière d'évaluation des politiques publiques, on veut bien aussi reconnaître un certain professionnalisme, de même qu'aux recherches entreprises sur les élites dirigeantes, non pas les politiciens, mais les groupes et grands corps aux contrôles des entreprises publiques, du monde des affaires, de la machine économique, de la "technostructure». Le politique est trop l'affaire de tous et un domaine trop brûlant pour le confier à l'examen de quelques ilotes ou experts autoproclamés (une centaine de chercheurs professionnels en tout).

16 À la différence de l'histoire, de l'anthropologie, de la sociologie, voire de la philosophie ou de la linguistique, qui ont produit quelques gourous ou intercesseurs médiatiques, les politologues n'ont pas pignon sur écran. Ils ne contrôlent pas les discussions théoriques publiques concernant leur objet. Ajoutons, à ce stade, un élément qui n'est 
pas négligeable et qui tient à l'influence des problématiques, des objectifs, des méthodologies et de la terminologie anglo-saxonne sur la science politique française depuis la seconde guerre mondiale. Aux États-Unis, la sortie de la guerre a été aussi une ouverture sur le monde à laquelle la discipline n'était pas préparée. Comme dans d'autres domaines, économiques notamment avec le plan Marshall, s'est opérée une reconversion d'une ampleur sans commune mesure avec ce qui a pu se dérouler en France. D'où une prospérité, soudaine et fragile à la fois, des recherches en direction des sociétés étrangères. Mais aussi un renouveau de la pensée scientifique, fortement enrichie par l'apport de savants étrangers, notamment d'Autrichiens et d'Allemands expatriés à la suite de la montée du nazisme pour partie. D'où des recherches empiriques d'un nouveau genre, que coiffèrent des réflexions épistémologiques et méthodologiques tout à fait neuves elles aussi.

En France, un peu empêtrés par les entrelacs de la tradition juridique, des perceptions "gouvernementalistes" (la science politique perçue et traitée comme "science de l'État »), et des débats théoriques (Rousseau ou Montesquieu, Tocqueville ou Durkheim, Weber ou Marx, pour schématiser), les politistes ont humé un peu d'air frais venant de l'Atlantique. Les spécialistes de la sociologie électorale ont sans doute glané, les premiers, du côté des études psychologiques du comportement (les behavioristes pour reprendre un mot sans grâce passé à la langue française), partant des choix électoraux, donc des techniques de sondage, mais aussi du côté de la personnalité politique, des acteurs sociaux. Ils ne sont pas les seuls, loin s'en faut.

Il est impossible de citer l'efflorescente production américaine, même d'en résumer les tendances. Je dirai seulement qu'elle a pesé sur la génération des années 1950, pour ce qui est des enseignants-chercheurs et sur celle des années 1960-1970 pour ce qui est des étudiants, les premiers assurant alors le relais au bénéfice des seconds, plus peut-être que la théorie marxiste des classes sociales ou que les paradigmes dépendantistes conçus en fonction des rapports économiques mondiaux. Non toutefois au point de remplacer les enseignements traditionnels, mais suffisamment pour influencer des contenus, orienter vers de nouveaux horizons, modifier des approches. Nul doute que les grands noms du développementalisme ou de la modernisation politique, du structuralo-fonctionnalisme, de l'analyse systémique ou du néo-patrimonialisme ont eu leurs épigones français. Sans qu'on puisse pour autant crier à la dépersonnalisation, à l'ethnocentrisme aveugle, à l'évolutionnisme béat ou à l'impérialisme culturel. Au fond, chacun aura butiné ce qui lui convenait le mieux. Et il n'est pas certain que les " nouveaux regards " portés par les politicologues français sur les systèmes et régimes politiques étrangers n'aient pas bénéficié de cet apport-là. Lire Talcott Parsons, surtout tel que critiqué par Anthony Giddens, Edward Shils et Samuel Huntington, replonger dans l'histoire par l'entremise de Robert Nisbet ou Charles Tilly, reprendre les propositions wébériennes touchant l'apparition du politique ou les études sur la différenciation structurelle telles que répercutées par différentes écoles d'OutreAtlantique n'entache pas ipso facto les travaux réalisés à Paris, Strasbourg, Lyon, Toulouse, Bordeaux ou Rennes.

Et le domaine étranger en effet dans tout cela? Intégré, de façon insensible, au champ comparatif du droit constitutionnel, sous l'appellation des «régimes politiques (contemporains)", il était surtout centré sur des grandes configurations institutionnelles, de longue tradition, soit proches du type Europe ou États-Unis, soit différentes, comme l'URSS et les « démocraties marxistes » (titre d'un cours enseigné 
dans les années 1950 par Georges Vedel à l'IEP de Paris). Avec quelques enseignements exotiques, surtout en direction de l'Extrême-Orient, de l'Amérique latine, par exemple. Peu sur l'Afrique et quasiment rien sur le monde arabe hors du Maghreb.

On explique ceci par la décolonisation et cette sorte de complexe ressenti vis-à-vis d'entités politiques exogènes que la France n'était parvenue ni à dominer ni à transformer. On sortait d'une histoire de pratiques politiques et méthodes de gestion de pays sous contrôle et il n'était guère facile de rebasculer en direction de pays devenus indépendants, d'autant qu'on tendait à juger leurs jeunes institutions à l'aune des critères de la vieille démocratie française (et ce alors que la colonisation avait conduit à nier les principes mêmes dont la France avait longtemps prétendu se réclamer). En pleine guerre d'Algérie (1954-1962) il n'était pas facile d'imaginer, rue Saint-Guillaume et moins encore place du Panthéon, fief de juristes traditionnellement plus à droite, un cours sur les régimes politiques arabes, sans que le contexte politique ambiant ne pèse sur les rapports exposant-auditoire. Souvenons-nous aussi des diatribes anti-nassériennes: l'Égypte étant accusée non seulement de soutenir diplomatiquement la cause algérienne mais de fournir en armes, matériels et subsides la résistance en Algérie même. Rappelons la folle équipée de Suez, dernier hoquet d'un impérialisme d'un autre âge dans laquelle s'est lancé le national-molletisme.

21 Notons que le premier manuel, fort original pour l'époque, sur les dits régimes arabes (rédigé par un historien spécialiste du domaine ottoman et un juriste internationaliste) date de 1968, c'est-à-dire six années après que le dernier pays arabe sous domination française se soit libéré. Mais signalons tout de même que dans les calculs qu'il a effectués par le biais de l'index analytique des livraisons des deux premières décennies (1950-1970) de la Revue française de science politique, Yves Schemeil révèle que dans les domaines d'étude abordés à travers les comptes-rendus d'ouvrages, le Moyen-Orient représente $13,2 \%$, soit $10 \%$ de moins que l'Extrême-Orient mais plus que l'Amérique latine et l'Afrique réunies.

En matière de pays arabes, le Maghreb - là aussi de façon coutumière en raison des liens (au double sens d'assujettissement et de relations antérieures) - a beaucoup plus retenu l'attention que le Machreq. Dans la première édition de Les régimes politiques des pays arabes à laquelle je viens de faire allusion, la répartition au sein de la troisième partie consacrée aux « Régimes locaux » comporte environ 50 pages sur le Maghreb, à peu près autant sur ce que les auteurs nomment «la mouvance égyptienne » soit l'Égypte, la Syrie, le Yémen, l'Irak, et l'équivalent sur le reste, les sociétés politiques dénommées "les indépendants ». Cet équilibrage d'apparence salomonienne recouvre des déséquilibres profonds. Ainsi, l'Égypte bénéficie de seize pages, autant que l'Algérie et à peine trois de plus que le Liban par exemple, donc de deux objets d'attention traditionnels du côté français. Ces pondérations sont, bien sûr, artificielles. Elles le sont moins si on applique le même genre de traitement à la littérature d'analyse au sein de laquelle on regroupera les productions journalistiques et scientifiques mais aussi les plaidoyers, témoignages et ouvrages d'autochtones publiés (et, pour une partie d'entre eux, traduits en français).

23 Ce que l'on constate, en France, tient à plusieurs aspects. D'abord, à la déflagration de 1952, qui sort un État-nation des cadres de l'analyse et des répertoires en usage. L'Égypte politique n'intéresse qu'à partir du moment où elle est "en mouvement ", selon le titre de l'ouvrage de Jean et Simone Lacouture qui date de 1958. Avec une exception toutefois, le livre consacré par Marcel Colombe à L'évolution de l'Égypte 
1924-1950, publié en 1951. Et ce mouvement, c'est, à l'évidence, l'armée des Officiers libres au pouvoir. Le point focal en est bientôt "Gamal Abdel Nasser et son équipe " auxquels G. Vaucher consacre deux volumes à la même époque (1959-1960). Avec des textes critiques, ceux d'Égyptiens qui ne partageaient pas l'idéologie du régime, qualifiée de populisme, ou qui récusaient ses pratiques d'encadrement, le régime monopartisan, l'autoritarisme, le culte du za'îm, entre autres. Les livres d'Anouar AbdelMalek, Égypte, société militaire (1961), de Ahmad. Abdul Fath', L'affaire Nasser (1962), de Hassan Riad, L'Égypte nassérienne (1964), ressortent de cette catégorie. Et on peut s'interroger sur l'accueil qu'a réservé l'opinion publique, le lectorat, en France, à ce type d'ouvrage. Combien de Français auront lu La philosophie de la révolution, dont une version française était fournie par le gouvernement égyptien et pour laquelle une traduction a été établie par la très officieuse Documentation française (Notes et Etudes documentaires), dès après la sortie de l'édition arabe (1954) ?

Ensuite, on observe que la part de la contribution académique est quasi nulle, par rapport aux enquêtes journalistiques ou aux essais, mémoires... La seule étude digne de ce nom est celle d'un Égyptien, sociologue, Anouar Abdel-Malek, déjà citée. À quoi on peut ajouter, en français, celle d'un autre sociologue, suisse celui-là, Jean Ziegler qui, dans sa Sociologie de la nouvelle Afrique (1964), consacre une partie à l'Égypte. Pour le reste c'est le désert occidental ajouté au désert oriental. On peut avancer une hypothèse, sans grand risque : l'enseignement français n'a pas produit de spécialiste susceptible de comprendre puis d'expliquer ce qui venait d'arriver au cœur du monde arabe.

Il faut tout de même signaler qu'une génération aurait-elle reçu les enseignements les meilleurs, aurait eu du mal à saisir ce qui se passait. Si l'on se reporte à l'époque, s'enchaînent sans discontinuer des phénomènes pour le moins inattendus: l'insurrection du Caire, le renversement de la monarchie, la disparition du système parlementaire et l'interdiction des partis, la mise à l'écart du général Neguib, l'arrivée aux commandes de militaires, puis cette révolution politique proclamée révolution sociale par la bouche de son leader, cela c'est pour l'intérieur. Et on était en droit de s'interroger sur la nouvelle légitimité qui pouvait bien sourdre de tout ce chambardement. Quel avatar nouveau du despotisme oriental avions-nous là ? Pour l'extérieur, ce n'était pas mal non plus: le coup d'éclat (après le coup d'état) de la nationalisation du Canal de Suez, suivi de la défaite diplomatique des anciennes puissances coloniales alliées à Israël. Les valeurs se trouvaient renversées. Avec les utopies tiers-mondistes qui fleurissaient, en Amérique latine, en Asie, avec la Chine, le Vietnam, le mouvement des non-alignés. Avec le panarabisme aussi, illustré par des projets d'union (Syrie, Yémen). Sans oublier la politique de bascule et le "passage » à l'Union soviétique. Autant de défis, de provocations qu'il était difficile à l'Occident de comprendre. Personne n'était préparé à cette série de chocs, au Conseil de la Révolution, au socialisme, à Bandoung, à Suez et personne, moins encore, n'était prêt à en décortiquer les causes.

26 La France, coupée de ses voies d'accès et sources d'influence traditionnelles (écoles chrétiennes, Ecole de Droit) après 1956, pouvait envoyer ensuite ses meilleurs journalistes (et Jean Lacouture en fait partie, de même que Claude Estier, auteur d'un petit ouvrage ambigu publié en 1965) mais aucun analyste patenté, enseignant ou chercheur. À lire les thèses préparées en droit, économie, science politique, ce n'est pas de ce côté-là qu'avaient été orientés les étudiants. L'université française avait produit 
des orientalistes, des islamologues, des arabisants, capables de commenter les textes anciens, de débattre des grandes époques de la civilisation arabe, de décrire les plus brillantes réalisations, intellectuelles, architecturales, de disserter sur le Califat, de disputer des points de doctrine religieuse qui avaient opposé autrefois de vénérables cheikhs. Mais il n'y avait pas de sociologue politique du temps présent ayant effectué un séjour au-delà de la Méditerranée et susceptible de rendre compte de ce qui s'y déroulait et qui, d'ailleurs, était proprement atypique.

Dernier élément, quelques commentaires ont bien été rédigés et ont paru dans des périodiques de réputation scientifique avérée, quelques thèses ont bien été entreprises. Mais, mis à part quelques articles et un livre sur le monde ouvrier (Céres Wissa Wassef et F. J. Tomiche, respectivement en 1973 et 1974), la dominante restait juridique, non politologique. Elle demeurait dans le droit fil de la longue influence du droit public sur le champ politique au sein des Facultés françaises, jusqu'à la création d'une agrégation de science politique (1971) au moins (et au CNRS jusqu'à la scission de la section «Sciences juridiques et politiques », soit jusqu'en 1983).

28 Jacques Robert et Yves Jouhaud, le premier dans la Revue de Droit public $\left(1965, \mathrm{n}^{\circ}\right.$ 5) et le second dans la Revue juridique et politique (1964) se sont livrés à l'exercice d'école, de type classique et par là même parfaitement maîtrisé, de lecture commentée des documents constitutionnels, en l'occurrence celui de 1964. On pourrait retrouver, je suppose, des développements du même genre, traitant de la constitution provisoire de février 1953 et de celles de 1956 et de mars 1958 (création de la RAU) ou des charte et proclamation de 1962.

29 Les articles ont un caractère parfaitement démonstratif. Le fondement de la légitimité, l'énonciation des grands principes, l'articulation des organes, la hiérarchie des pouvoirs, la prépondérance de l'Union socialiste, tout y est, de façon formelle, sans grande référence a la réalité. On nous décrit des mécanismes institutionnels, non des rapports de force. On évoque des référents à caractère légal, sans dévoiler la nature d'un régime. On décrit des structures, des mécanismes, non des dynamiques, des mutations et des conflits. Or, l'étude du politique c'est tout de même la mise en rapport des uns et des autres! De plus, l'optique constitutionnaliste et le juridisme du commentaire inscrivent implicitement des textes normatifs étrangers dans une tradition qui n'est pas la leur, les font apprécier par rapport aux modèles occidentaux, comme produits quasi naturels d'un "contrat social» ou de compromis passés entre gouvernants et gouvernés, alors qu'ils répondent à d'autres fins, rendent compte d'autres rapports de force, sont les fruits d'une volonté unilatérale, les instruments d'exercice d'un pouvoir. On est encore dans la lignée des thèses soutenues dans les Facultés de Droit françaises par des chercheurs égyptiens durant l'entre-deux guerres, à l'image de celle de Amin Osman (Le mouvement constitutionnel en Égypte et la constitution de 1923,1924).

$30 \mathrm{Au}$ moment où Nasser meurt, ce que l'on pourrait appeler la couverture politologique de l'Égypte n'est guère mieux assurée qu'avant. Jean Lacouture ramasse ses informations et propose ses interprétations du leadership dans une sorte d'éloge funèbre (1971). On traduira ensuite en français et assez rapidement après leur édition arabe, les ouvrages d'un des proches du président, Mohamed Heikal. Du côté universitaire, Maurice Duverger, qui a engagé quelques-uns de ses étudiants à systématiser les méthodes mises en œuvre par lui et à vérifier ses hypothèses sur les partis politiques, les syndicats et groupes de pression, sur les institutions politiques 
aussi, en les appliquant à différents régimes de la planète, dirige puis préface la thèse de notre collègue Afaf Mahfouz soutenue en 1968 et publiée à Paris en 1972 sous le titre Socialisme et pouvoir en Égypte. Thèse de science politique qui avait été précédée ou suivie par celles de Ibrahim Saleh (Le régime politique de la RAU et la notion de démocratie, soutenue à Paris toujours en 1966), Ali al-Samman, Saïd Zulficar, par exemple.

31 Entre temps, ont paru les deux grands ouvrages de politologie de la période, et qui ne sont ni l'un ni l'autre non plus l'œuvre de "politistes", mais d'hommes difficilement classables, l'un islamologue, l'autre sociologue. L'Égypte. Impérialisme et révolution est sorti des presses en 1967. Inutile de faire le panégyrique d'un ouvrage lu et relu, et dont la fréquentation a été conseillée et quelquefois imposée à des étudiants ou apprentis chercheurs. Le livre a fait l'objet de critiques. On a prétendu qu'il n'était pas facile à lire, trop touffu, entaché d'erreurs. Ce qui expliquerait, peut-être, pourquoi il ne figure pas dans la bibliographies des deux éditions des Régimes politiques arabes (celles de 1968 et de 1990) car on ne peut imaginer que les auteurs des parties « Égypte » l'ignorent ; ce qui serait pour le moins ahurissant: lorsque l'édition française est si pauvre sur une société politique si riche, il est mal venu d'écarter une oeuvre de ce calibre. On a là, en effet, 700 pages sur l'Égypte prénassérienne, dont près de la moitié portent sur la phase 1919-1940 et qui constituent un remarquable pendant au Maghreb entre deux guerres du même auteur. Jacques Berque a compris que la seule manière d'expliquer 1952 c'était de retourner un siècle en arrière, 80 ans très exactement. Et de reprendre le périple en marquant l'arrêt à chaque événement d'importance, devant chaque contradiction révélée, chaque tension explicitée, chaque crise, de 1907, de 1919, 1929..., les avatars de ce que J. Berque nomme la "variation historique». Ce retour sur l'histoire, à partir d'une technique de la diffraction et dans un langage qui est le sien, c'est cela qui lui permet de reconstruire cette relation dialectique de la dépendance et de la libération ${ }^{2}$.

Autre ouvrage de poids, Idéologie et renaissance nationale. L'Égypte moderne, paru en 1969, épouse un parcours semblable, avec des techniques totalement différentes. Anouar Abdel-Malek y reconstitue, de l'intérieur, un processus unitaire grâce auquel une communauté politique s'est agrégée et autonomisée. L'étude fait partie - peut-être plus que Égypte: société militaire, plus jeune de sept années et plus volontiers cité par les politistes - de ces introductions à la connaissance d'un pays dont il paraît difficile de faire l'économie. Toutes proportions gardées, l'ouvrage collectif réalisé par l'équipe aixoise et publié au CNRS en 1977, L'Égypte d'aujourd'hui, 1805-1976, adopte une formule assez proche - beaucoup plus didactique que celles de Jacques Berque et Anouar AbdelMalek, plus systématique donc moins intuitive mais, par là même, ne possédant pas la même tonicité -, celle de la quête historico-politique des causes. Le seul regret que l'on peut formuler est que les politologues en soient absents. D'ailleurs où sont-ils? Qu'ontils fait depuis?

33 Ne nous livrons pas à une recension oiseuse des travaux publiés, en séparant purs essais, recherches proprement dites, thèses universitaires, etc., en décomptant ouvrages et articles... Et contentons-nous de faire remarquer que le renouveau de la recherche politologique française concernant l'Égypte est lié à trois «faits »appelons-les comme cela.

Le premier, c'est la part prise par les Égyptiens eux-mêmes dans le discours scientifique (en français) sur l'Égypte. On a mentionné les travaux d'Anouar Abdel-Malek. Il faut dire l'impact de deux auteurs (trois puisque le premier mentionné est un nom de plume commun à deux personnes), Mahmoud Hussein et Samir Amin. Plusieurs courants, où 
se retrouvent des intellectuels de diverses gauches, en rupture avec le régime ou, à tout le moins, critiques explicites du nassérisme après la défaite de 1967, se sont exprimés à travers eux.

La lutte des classes en Égypte, publiée une première fois en 1969 et republiée depuis (en 1971 et 1975) et traduite en anglais (1973) a secoué l'académisme tiers-mondiste déjà installé. L'ouvrage prétendait livrer les clés d'une lecture socio-politique de l'Égypte, en reprenant, et en l'affinant en même temps, la méthodologie marxiste. Nous avions là, soudain, et dans la ligne du grand remue-ménage idéologique et scientifique de 1968, la démonstration que les classes sociales, les fractions de classes, avec leurs alliances et renversements d'alliance, entre éléments de la «bourgeoisie nationale " notamment, pouvaient œuvrer pour la compréhension d'une société du Tiers-Monde. Certains ont délibérément ignoré (ou refusé) ce néo-marxisme; d'autres l'ont condamné comme tout aussi systématique que l'archéo-marxisme. Mais le débat a été porté par une minorité agissante sur le terrain académique, celui de l'enseignement et de la recherche; et ce au moment où s'effectuaient diverses relectures de Marx, sous l'influence de Louis Althusser (Pour Marx, 1965, Lire le Capital, 1966) ou Nicos Poulantzas (Pouvoir politique et classes sociales de l'État capitaliste, daté de 1970) par exemple. Et il serait intéressant de relever les traces que le livre de Mahmoud Hussein a laissées dans les cerveaux en dépit de sa démonétisation ultérieure, de repérer aussi comment l'Égypte autoritaro-populiste de l'époque devenait une sorte de modèle où lire « l'avenir » du monde arabe, et, qui sait ?, du Tiers-Monde, dans leur ensemble.

Les travaux de Samir Amin, économiste d'origine égyptienne, (L'économie du Maghreb... 1966 ; Le développement inégal : essai sur les formations sociales du capitalisme périphérique, 1973 ; La question paysanne et le capitalisme, 1974, L'impérialisme et le développement inégal et La nation arabe : nationalisme et lutte des classes 1976) ne prenaient pas l'Égypte comme objet. C'étaient plutôt le Maghreb ou l'Afrique Occidentale qui retenaient l'attention. Mais l'interprétation, reproduisant au niveau du Moyen-Orient une lutte des classes, appliquant au niveau mondial une théorie de la dépendance forgée à partir de cas latinoaméricains, ouvrait tout à coup sur des perspectives planétaires, des discussions théoriques, un domaine comparatif auxquels les chercheurs n'étaient plus habitués.

Ceci nous amène à une double constatation : la réinscription de l'Égypte dans un champ théorique et analytique de grande ampleur a été le fait d'Égyptiens dont la spécialité n'était pas la science politique; ils n'ont guère été suivis sur ce terrain par leurs homologues français, qui ont préféré les voies plus orthodoxes - et moins dangereuses - de l'empirie. Le résultat, c'est que, jusqu'à ces toutes dernières années, aucun politiste en France n'est devenu la référence lorsqu'il s'est agi ne serait-ce que de commenter une actualité politique pourtant riche en interrogations de tous ordres. L'université française n'a pas produit l'équivalent d'un John Waterbury, par exemple. Et c'est, une fois de plus, un chercheur d'origine égyptienne, Cérès Wissa-Wassef qui, au Centre d'études et de recherches internationales (CERI) a proportionnellement le plus écrit sur les événements survenus au pays des pharaons.

Le second fait pourrait tenir, comme partout ailleurs dans le monde arabe, au renouveau islamique. N'entrons pas dans les débats quintessenciés et acrimonieux en cours pour savoir comment il faut le nommer, quelles formes il prend. Disons simplement que la politisation de ce qui était le secteur de la foi, avec en regard la pression exercée sur le politique par le code religieux, que ce double mouvement a profondément modifié le paysage politique, mais aussi la culture sociale, les rapports 
État-citoyens... Nommons cela effet iranien, désidéologisation ou désaffection vis-à-vis de l'État national (impuissant à répondre aux attentes sociales et à opérer la redistribution économique sur des bases égalitaires), militantisme islamiste comme nouvel espoir pour sortir du sous-développement ou de l'influence US, peu importent les motifs et manifestations, pour ce qui nous concerne. Ce qui doit nous retenir c'est le recours aux (donc le "retour» des) politologues frottés d'islamologie, re-légitimés soudain par ce que ce qui se passait en Orient, dans le monde musulman, dans les pays arabes et qui, pour ce dont nous parlons, l'Égypte, échappait totalement aux politologues, qu'ils soient de formation académique classique ou le produit de cette nouvelle génération que Jean-Pierre Péroncel-Hugoz avait appelé il y a quelques années déjà les «nouveaux orientalistes ».

39 Les études d'Olivier Carré, de la fin des années 1970, sur la légitimation islamique des socialismes arabes, et qui allaient évoluer ensuite - après le renversement du shah d'Iran et l'installation de la mollarchie - en direction d'autres radicalismes, prenaient un sens, acquéraient en quelque sorte un statut académique, une valeur médiatique, imprévus. En 1979, l'imam Khomeiny provoquait, dans les consciences occidentales, le même type de choc que l'arrivée des Officiers libres en Égypte 27 ans auparavant.

Peu de personnes, y compris des spécialistes de l'Iran - pensons à Paul Vieille en France, Myron Zonis aux USA - avaient soupçonné un bouleversement de ce type, et pensé que le chiisme considéré plutôt comme passéiste pouvait enfanter une révolution politique, car c'est bien de révolution qu'il s'est agi. Mais Nasser, c'était le socialisme arabe, le tiers-mondisme, l'insurrection politique et morale contre l'Occident, où l'islam tenait peu de place, du point de vue des commentateurs étrangers. Avec Khomeiny, la religion envahit le champ à tous les sens du terme. Pour l'heure, il s'agit de l'Iran, non de l'Égypte, mais pour deux ans seulement. L'assassinat du président Sadate, au nom d'une interprétation puritaine et extrémiste de l'islam, nous ramène sur les bords du Nil, en fanfare. L'Égypte, de 1970 à 1981, avait rassuré. Elle était retournée progressivement dans sa mouvance traditionnelle, celle de l'ouest, après le renvoi des Soviétiques, les accords de Camp David, la politique économique de l'infitah, même si le régime ne réalisait pas l'ouverture démocratique équivalente à laquelle on l'invitait. Au fond, elle apparaissait comme une nation musulmane que recouvrait un État laïc, ou peut s'en faut. L'attentat a remis en cause cette image à laquelle on se laissait aller outre-Méditerranée et outre-Atlantique, ne serait-ce que parce que c'était celle que l'on avait envie de voir. Et la liaison chiisme iranien-radicalisme islamique (qu'il soit sunnite ou non) s'est opérée à ce moment-là.

41 D'où cette seconde vague d'articles et d'ouvrages de la décennie 1980-1990, qui recentrait sur le Caire et disons Assiout (puis sur l'ensemble de l'Afrique du Nord à la suite des développement algériens et tunisiens), ce que ceux de la vague précédente avait aidé à focaliser sur Téhéran et Qom. Et là, pas question de laisser des journalistes expliquer l'événement, le décrire peut-être, l'expliquer sûrement pas. On sortait du politique tel que conçu comme une préconnaissance générale. Il fallait recourir à des gens dont le savoir permettait de rendre compte de cette brutale - et estimée dangereuse - repolitisation par le religieux.

Une catégorie de politologues a reçu alors l'ondoiement. Elle s'est fait reconnaître d'utilité publique. Si Yann Richard (dont le Chiisme en Iran date de 1980 et l'Islam Chiite. Croyances et idéologies vient de sortir) a enfin pu se faire entendre au-delà du cercle des iranologues, si les études d'Olivier Roy ont eu le rayonnement qu'elles ont eu à la suite 
des résistances à l'occupation soviétique de l'Afghanistan, la thèse de Gilles Kepel publiée sous forme d'ouvrage en 1984, le livre d'olivier Carré sur la pensée mystique et révolutionnaire de Sayyid Qutb paru la même année, sont aussi sortis du ghetto habituel des spécialistes et ont atteint un large public. On a pu croire que la politologie islamologique avait gagné en statut et atteint le niveau d'autres branches, de la sociologie électorale ou de celle des organisations par exemple. Mais il n'est pas certain que cette promotion soit assurée de façon irréversible.

La troisième donnée de ce retour de l'intérêt pour l'Égypte ne vient pas de l'Égypte ellemême et n'est pas liée aux perceptions que l'opinion française a pu se faire de l'islam. Elle tient à une meilleure connaissance du monde arabe ayant passé par un contact très direct, (et de trois années minimum pour ce qui est des chercheurs), avec la société qui se trouve placée justement en son centre. Depuis que le CEDEJ existe, soit depuis une vingtaine d'années, ont séjourné entre ses murs environ une trentaine de chercheurs français. Un tiers d'entre eux était déjà partie prenante d'institutions d'enseignement ou de recherche avant de se rendre au Caire; un tiers est entré au CNRS après être passé par l'Égypte ; le dernier tiers est actuellement sur place.

Ajoutons que ce qu'ont reçu ces chercheurs et apprentis chercheurs ne consiste pas seulement en une formation complémentaire après des études de sciences sociales et de langue effectuées en France même le plus souvent mais implique des moyens de travailler et de s'exprimer; les thèses de Gilles Kepel (Le Prophète et Pharaon...) et de Pierre Mirel (L'Égypte des ruptures: l'ère Sadate de Nasser à Moubarak, parue en 1982), toutes deux rédigées au CEDEJ, des ouvrages, un périodique, en fournissent la preuve... La politologie n'en est pas absente, comme en témoignent les Actes de deux colloques (Études politiques du Monde arabe. Approches globales et approches spécifiques d'une part, et Démocratie et démocratisations dans le monde arabe de l'autre) ainsi que diverses contributions à la nouvelle revue Égypte/Monde arabe et trois numéros spéciaux de périodiques, "Égypte. Recompositions" (Peuples Méditerranées, 41-42, octobre 1987mars 1988), «Égypte 1990. Enjeux de société » (Maghreb-Machrek 27, $1^{\text {er }}$ tr. 1990), et «Égypte : années 1980 » (Revue Tiers Monde 121, $1^{\mathrm{er}}$ tr. 1990) tous trois pilotés par Alain Roussillon, devenu en quelques années le politologue français spécialiste de l'Égypte.

\section{La sociologie politique nord-américaine : une position particulière}

Commençons par des remarques sur les études avant d'énumérer les centres d'intérêt successifs autour desquels elles s'organisent.

Première constatation : en général, les études nord-américaines concernant l'Égypte, celles menées aux USA ne diffèrent pas de celles concernant les autres ensembles politiques de la région, le Croissant fertile (Syrie, Irak, Jordanie, Palestine) le Maghreb, ou la Péninsule arabique, sans oublier le Soudan.

Donc, les paradigmes - modèles ou ensembles de concepts types construits pour rendre compte de phénomènes - qui ont été utilisés, sont les mêmes pour l'Égypte que pour les autres pays. Toutefois, l'Égypte bénéficie d'une sorte de position privilégiée, en ceci qu'elle a donné lieu à une littérature beaucoup plus abondante que n'importe quelle autre communauté politique de la même ère culturelle, mais aussi que les analystes ont eu quelque peine à lui appliquer sans nuances les explications en termes de 
développement, de modernisation ou de différenciation politique, par exemple, élaborées à partir de cas africains, asiatiques ou latino-américains. Comme si les "logiques spécifiques» du "champ " ou de "l'espace social » ou du "système » égyptien interdisaient de l'assimiler aux autres sociétés censées présenter des caractères analogues.

La production académique touchant l'Égypte est de même poids que celle relative au reste du monde arabe. Surtout, il y a beaucoup plus d'ouvrages consacrés à l'Égypte seule qu'à aucun autre pays. Beaucoup des travaux réalisés englobent l'ensemble «moyen-oriental» ou «monde arabe»; peu, hors de l'Égypte encore une fois, sont dévolus à un seul cas. On pourra expliquer ceci par le poids démographique, par la centralité géostratégique, par l'ancrage historique, le passé politique, aussi par le fait que, depuis la seconde guerre mondiale, l'Égypte est un élément essentiel dans la politique extérieure des États-Unis, en Méditerranée, en Afrique, en terre arabe, et un facteur déterminant dans la protection d'Israël, objectif majeur des USA, pour l'ensemble de la région. Il faut aussi avancer le poids spécifique des expériences politiques égyptiennes et leur importance pour la communauté scientifique ${ }^{3}$.

Tout ceci est sans doute vrai. Il y a tout de même, là aussi, un "effet Nasser " indéniable. Comme l'Europe, les États-Unis ont été pris à froid, et par deux fois : lors de la révolution tout d'abord, puis lorsque Nasser a rejeté le chantage américain au barrage d'Assouan et a requis l'aide soviétique. Mais, à la différence de la France, ils ont réussi à mobiliser des moyens, à former des gens, à orienter un enseignement, pour tenter de comprendre ce qu'il leur était advenu. Et ce qui leur était advenu se situait justement au Caire. La masse toute relative d'articles et d'ouvrages publiés mais aussi de thèses, d'essais - auxquels il faut ajouter les rapports multiples rédigés par les diplomates, conseillers, consultants, experts et autres - cette masse s'explique par l'intérêt porté par une société à une autre, intérêt pas du tout dégagé des contingences mais cela existe-t-il, l'intérêt scientifique pur?

Ajoutons que les États-Unis possèdent en Égypte des bases académiques, que chacun connaît: une université centenaire et deux centres de recherche, plus des organes d'appuis, Ford Foundation, Programme Fullbright, par exemple. L'Université américaine du Caire n'est pas une petite institution, ni en poids ni en réputation. Quelques évidences fondées sur des données chiffrables en témoignent.

51 En 1990-91, le Département d'Économie et de Science politique a accueilli dans la branche science politique, à tout prendre autonome, 220 étudiants dont 190 préparent (en 4 ans) une licence; les trente autres étant inscrits dans un master programme comportant l'obligation de rédiger une thèse (aucune préparation doctorale, Ph.D, n'étant assurée à ce jour). On a offert à la rentrée 1991 à ces étudiants 18 cours par semestre, au sein desquels ils ont sélectionné un maximum de 5. S'il n'y a qu'un cours de sociologie politique sur l'Égypte proprement dite il n'y en a qu'un aussi sur les USA. Chaque semestre est offert un cours de politique comparée sur les systèmes politiques du Moyen-Orient. Et dans de nombreuses matières, la part réservée à l'Égypte peut être considérée comme importante. De plus, un enseignement nouveau va être créé sur la politique extérieure de l'Égypte. Une précision: AUC n'a pas imposé sa version d'une science politique quantitative appuyée sur les mathématiques et statistiques qui sont considérés dans d'autres universités (aux USA) comme le nec plus ultra en ce domaine.

On pourra objecter qu'il s'agit d'enseignements destinés à des Égyptiens. Ladite objection peut être doublement levée. En premier lieu, il n'y a pas que des étudiants 
égyptiens, puisque 80 étudiants d'universités américaines viennent effectuer une année académique au Caire (cette disposition est partie intégrante de leur cursus) et que figurent des enseignements de science politique. Avec ceci de particulier qu'ils ont beaucoup plus de chance que leurs collègues restés aux USA de choisir des matières ayant le Moyen-Orient comme point d'application, et la chance tout court de se trouver en milieu dit « indigène ». En second lieu, on ne peut pas dire que la «reproduction » ne fonctionne pas, que AUC ne participe pas d'un processus d'acculturation, qu'elle n'autorise pas une population intellectuelle étrangère à assimiler un savoir. L'enseignement est effectué en anglais, en partie par des enseignants d'origine américaine (même si certains comme Walid Kazziha sont naturalisés). Des voies sont ouvertes, notamment pour les études doctorales, avec les grandes universités d'OutreAtlantique, offrant des bourses mais aussi une chance de se confronter avec d'autres méthodes, de s'initier à la recherche, de choisir des terrains d'étude en vue d'une thèse, différents de ceux dont on est issu, donc de s'ouvrir sur d'autres perspectives, sans parler du fantastique soutien logistique des campus et bibliothèques. Une recension systématique des doctorats de science politique obtenus aux États-Unis par des étudiants égyptiens devrait le confirmer ; les chiffres existent et sont accessibles. J'ai personnellement constaté, surtout après que les accords de Camp David aient été signés, que des filières existent entre l'Égypte et les USA, qu'un réseau s'est constitué, que la plupart des politologues en poste aujourd'hui au Caire, à l'Université ou dans les instances de recherche, ont effectué des séjours dans des institutions homologues en Amérique, quand ils n'y ont pas rédigé ou soutenu leur thèse. Il suffit de se rendre aux congrès annuels de la Middle East Studies Association (dont on a fêté le $25^{\text {ème }}$ anniversaire en novembre 1991, à Washington DC et le passage au-delà du $2000^{\mathrm{ème}}$ membre), pour être témoin de la participation égyptienne régulière à ces grands raouts cosmopolites. Tout ceci n'est pas propre à l'Égypte. Il y a eu, avant la chute du shah, des milliers d'étudiants iraniens dans les universités, de la côte ouest essentiellement. Il y a encore des centaines d'étudiants issus d'Arabie Saoudite et des autres micro-États de la péninsule arabique. Cela participe d'une sorte de mondialisation de la culture politologique, dont les universités US sont les amplificateurs et dont la langue anglaise est le véhicule.

53 J'ai parlé d'AUC, il faut y ajouter le Social Science Research Center, incorporé à l'université et que dirige un sociologue d'origine égyptienne ayant longtemps enseigné aux ÉtatsUnis (Saad Nagui). Et l'American Research Center in Egypt, fondation autonome, qui n'est pas un centre de recherche à proprement parler puisqu'il n'a pas de programmes spécifiques, ne coordonne pas des études, ne lance pas de projets portant son sigle (ARCE). Mais qui accueille des chercheurs indépendants, venus travailler dans le cadre d'une recherche (thèse, article, entre autres) avec des fonds propres, qui a tout de même des moyens non négligeables, une bibliothèque, notamment, et qui gère un séminaire annuel, etc. Et qui a eu, ne l'oublions pas, pour directeur un politologue d'origine libanaise, Ilya Harik, un des meilleurs connaisseurs de l'Égypte immédiatement contemporaine. Nous allons y revenir.

54 Enfin, précisons que tout ceci est complété par des éléments divers, constituant un dispositif typiquement américain, où l'État joue un rôle moindre que la plupart des grandes universités et que les fondations, privées les unes et les autres. Quand on considère le saut qualitatif réalisé, depuis un demi-siècle, c'est assez confondant. Souvenons-nous, que, après la deuxième guerre mondiale, les États-Unis étaient littéralement « sortis dans le monde » après deux décennies de repli sur soi et qu'ils n'y 
avaient compris goutte. Les systèmes politiques du Japon qu'ils occupaient, de l'Europe de l'Ouest qu'ils libéraient, de l'Europe de l'Est passant sous contrôle communiste et reproduisant le modèle du parti unique, de même que la Chine que Tchang Kaï Chek devait abandonner à Mao, les régimes du Tiers monde ou ce que l'on allait nommer tel, étaient terrae incognitae: mondes d'institutions étranges et univers de cultures politiques insaisissables.

Il y a toute une littérature, une filmographie à établir aussi, sur la manière dont les Américains ont ressenti une succession de chocs à la limite du traumatisme, avant de se dessiller les yeux. Et dont ils se sont remis, comme il a été suggéré à propos du nassérisme, en « mobilisant ». En dotant les fameuses area studies, études des différentes aires culturelles au sein des universités. En permettant aux grosses fondations, Carnegie, Ford, Rockefeller, d'en faire autant. Et le nombre d'universitaires spécialistes du Moyen-Orient venus en Égypte depuis la guerre est impressionnant. On ne peut en faire le décompte exact mais tous les auteurs connus ont eu l'occasion d'effectuer en Égypte un séjour dépassant une année en moyenne, y compris des spécialistes comme William Zartman dont l'objectif n'est pas l'Égypte mais le Maghreb, l'Afrique. Leonard Binder, l'enfant terrible de la politologie isiamologique, a effectué un premier séjour au début des années 1960, sous l'égide du Social Science Research Council dont sont sortis des essais sur l'idéologie nationale, le recrutement politique (1964 et 1966) bien avant le fameux (admiré et honni) In a moment of enthusiasm. Political power and the second stratum in Egypt (1978). Depuis, le courant ne s'est pratiquement pas interrompu. En 1991-92 ce sont Raymond Baker et peut-être John Waterbury, qui en seront à leur énième séjour, qui sont invités (pour un semestre ou une année) à $A U C$, où Raymond Hinnebusch, Robert Bianchi ont passé eux-mêmes quatre années pleines avant d'enseigner aux USA, où ont séjourné Clement Henry Moore, Allan Richards, Robert Springborg et d'autres.

Selim Nasr, politologue d'origine libanaise, préside aux sélections du «Concours de Recherches et d'Études en Science Sociales » (MERC) de la Ford Foundation dont il est le nouveau responsable, démontrant que, quarante ans après, l'Amérique met encore d'importants moyens au service de la recherche en sciences sociales et humaines sur le monde arabe, en dépit des concerts de lamentations entendus ici ou là. Elle assure une formation sur place sans comparaison avec aucun autre pays étranger.

Venons-en aux centres d'intérêt, aux matières et domaines qui ont retenu les politologues US depuis les années $1950^{4}$.

Ce dont on dispose, pour qui veut se documenter sur l'Égypte du «tournant », celle du renversement de la monarchie et de la montée de ce qu'on prend déjà l'habitude de nommer le nassérisme, tient, pour ce qui est de l'édition en langue anglaise, en peu d'ouvrages, ceux de 'Abdu al-Futuh Radwan (Old and new forces in Egyptian education, New-York, 1951) pour ce qui est de la culture; Charles Issawi qui a résidé en Égypte (Egypt: an economic and social analysis, 1947, mais surtout Egypt at mid century; an economic survey, Londres, 1954) puis, éventuellement, David Landes (Bankers and pashas... Cambridge, Mass., 1958) pour l'économie ; Jakob M. Landau (Parliaments and parties in Egypt, New-York, 1954) pour l'histoire politique récente ; E. Lengyel (Egypt's role in world affairs, Washington, 1957) pour les relations internationales. Petit bagage on le voit, bien que de qualité, à quoi viennent s'ajouter quelques essais journalistiques.

59 La première vague d'assaut n'est pas à proprement parler made in US. Elle est menée par un spécialiste du Moyen-Orient, d'origine grecque et de nationalité britannique mais qui enseigne alors la science politique à l'université d'Indiana aux États-Unis : 
Panayotis Vatikiotis, et qui publie en 1961 un livre dans le droit fil des interrogations du moment. The egyptian army in politics pose bien le problème central auquel chacun pense : est-on en présence d'une révolution initiée par les militaires, selon le modèle turco-iranien des années 1920, ou d'un bonapartisme précurseur d'une dictature ? P. J. Vatikiotis va plus loin. Il pose la question dès son sous-titre : tient-on avec l'entrée des militaires dans la vie politique, "un modèle pour les nouvelles nations" (a pattern for new nations)? Ce à quoi répondent les études d'Eliezer Béeri (Army officers in arab politics and society, 1970) ainsi que les ouvrages d'Amos Perlmutter, un israélo-américain qui a été commandant dans l'armée israélienne et doit savoir de quoi il parle, à travers son livre: Egypt the pretorian state, et de Ellenkay Trimberger qui date, lui, de 1970 (Revolution from above...). Autre "étranger américain » à entrer en lice, Nadav Safran avec Egypt in search of political community (1961), centre son ouvrage sur la société politique, le rapport entre homogénéité sociale et nouvelle élite au pouvoir. Ce que l'on peut situer sur un terrain proche de celui traité par George Gardner dans une thèse, soutenue la même année - 1961 - sur la transition entre tradition et modernité5. Et si l'on remonte quelque peu en arrière, on trouve un autre pivot autour de quoi ramasser les interrogations. Il est fourni par Morroe Berger et son Bureaucracy and society in modern Egypt qui remonte à 1957 et qui, là aussi j'emprunte au sous-titre, est une étude des hauts fonctionnaires (high civil service). Enfin, notons une série d'analyses liées au développement économique, telles que soulevées par Frederick H. Harbison et Ibrahim A. Ibrahim dès 1958 (Human resources for Egyptian enterprise), la plupart prônant volontiers l'industrialisation comme solution miracle, ou évaluées par un historien de l'économie, déjà cité, Charles Issawi, dont Egypt in revolution ; an economic analysis paraît en 1963.

On a là quatre points d'ancrage, l'unité de la communauté politique ou de la sociéténation, deux forces politiquement imposantes : l'Armée et la Haute administration et la capacité d'une économie à assurer sa nécessaire transformation. Ces thèmes ou objets d'analyse, on va les retrouver non seulement dans le reste du monde arabe, en tout cas en Irak et Syrie mais aussi en Algérie, au Soudan, ultérieurement, et dans le reste du Tiers monde, en Amérique latine, en Afrique, là où les fameux régimes prétoriens font florès. Il en sortira une espèce d'idéal-type, avec un début de théorisation pas toujours convaincant. Mais on peut dire que c'est grâce aux Officiers libres que s'est développée une recherche d'ampleur inaccoutumée, sur la place de l'Armée dans les nations, le rôle des militaires dans la politique. De ce point de vue, l'Égypte peut être considérée comme centrale. Elle pourrait l'être aussi pour les enquêtes de sociologie des groupes, des organisations. La trace de la bureaucratie conçue comme force susceptible d'exercer sinon des pouvoirs autonomes, en tout cas des influences au sein des appareils d'État, avec ses clans et ses alliances, mais aussi comme composante sociale différenciée, cette trace nous la retrouverons vingt ans plus tard avec Nazih Ayubi, auteur en 1980 de Bureaucracy and politics in contemporary Egypt et plus près de nous, par le biais de l'ensemble des contributions rassemblées par Monte Palmer, Ali Leila et le Dr. El-Sayed Yassin sous le titre The Egyptian bureaucracy (1988).

61 Pendant plusieurs années, les recherches tournent autour de ces axes ${ }^{6}$. Avec, en arrière plan, tout l'appareil conceptuel du développementisme, les références structurofonctionnalistes, qu'il faudrait intégrer à la description, ne serait-ce que parce que, à partir de cette première période où les enquêtes sur l'Égypte ont enrichi en partie la problématique politologique US, l'Égypte, peu ou prou, ne va plus servir que de champ d'expérience pour vérifier des hypothèses formulées ailleurs, outre-Atlantique pour 
l'essentiel. Et l'Égypte est insérée, au même titre que le Nigéria, l'Ouganda, le Maroc, l'Indonésie... dans le dispositif. À l'exception, peut-être, des analyses en termes de leadership où, là encore et toujours, la personnalité de Nasser pose quelque problème aux analystes.

Le cinquième et nouveau point de fixation c'est, en effet, la présence à la tête de l'État égyptien d'un leader charismatique répondant à une des catégories wébériennes ${ }^{7}$. Du temps du vivant du président, les véritables études ont été rares. À sa mort, les spécialistes recomposent un régime marqué par la personnalité de son leader et le passent au filtre de leurs hypothèses. Il leur faut répondre à la question classique : « Qui gouverne?", c'est-à-dire qui a gouverné et comment, au Caire, jusqu'en 1970. Si l'ouvrage de l'ancien ministre britannique Anthony Nutting, Nasser (1972), fait un peu penser à ce qu'écrit alors Jean Lacouture du côté français, l'essai de Hrair Dekmejian est d'un autre tonneau. Egypt under Nasir publié dès 1971, sous-titré " une étude de la dynamique politique ", est le produit d'une méthode par laquelle est mise en relief la relation entre une société et celui qui en est le chef incontesté, autrement dit la légitimité politique. Sept ans plus tard, Panayotis Vatikiotis tentera, avec Nasser an his generation, un bilan rétrospectif d'une société dont devaient émerger une nouvelle classe politique et un homme porteur d'espoirs messianiques. Entre temps, différents états de la période auront été dressés, dans des cadres académiques, par le biais de thèses notamment, aux USA mais aussi à Londres par des étudiants arabes et quelquefois égyptiens, tels Mohamed Abdel Halim El-Asram (New-York University, 1972) et Mohamed Bashir Hamid (Université de Sussex, 1973).

63 Sixième objet d'étude supplémentaire : après les leaders, des élites (et, non pas les élites) et les fractions d'essence pas seulement politiques. La mode, au milieu des années 1970, est de recenser non plus le sommet de la pyramide (c'est l'image qui s'impose d'ellemême) socio-politique mais les degrés qui sont directement inférieurs. Robert Sprinborg (1975) traite du cas égyptien et des modes d'association de l'élite politique dans l'ouvrage collectif que dirige George Lenczowski (Political elites in the Middle East, 1975) et Sharough Akhavi remplit la même fonction en abordant « l'élite patrimoniale » au sein d'un autre ouvrage piloté par Frank Tachau, et publié la même année (Political elites and political development in the Middle East). On nage aussi en plein néopatrimonialisme, clientélisme, autres agrégats et formes associatives. Robert Springborg propose de transformer le terme de chilla (ou shilla) en concept opératoire pour l'Égypte et au-delàs. Que l'on sache seulement, et le livre de Leonard Binder cité (l'étude de la "seconde strate " des élites égyptiennes) en témoigne, que l'on est à la recherche de ceux qui influent sur les décisions, qui contrôlent pour partie la machinerie étatique ou se situent à des niveaux, ou à des jonctions, détiennent des clés. L'enquête de Clement Henry Moore sur les ingénieurs (Images of development. Egyptian engineers in search of industry, 1980), elle, est une véritable étude anthropologique d'un milieu professionnel, dont les attentes en termes d'emplois ne peuvent être satisfaites pour la plupart, d'un milieu différencié comportant une hiérarchie de statut et de prestige et dont les rapports entretenus avec le politique se ressentent. Il s'agit bien encore d'élite, celles des diplômés, mais d'une élite fragmentée, décalée dans sa majorité.

64 Septième secteur, assez proche du précédent, celui des groupes d'intérêt. Robert Springborg, déjà nommé, a commis un article en 1978 sur « les syndicats professionnels dans la politique égyptienne de 1952 à $1970^{\circ}$ ». Et Robert Bianchi, à travers l'examen des 
phénomènes corporatistes, a observé l'ensemble politique égyptien du point de vue des groupes d'intérêt, (cf. Unruly corporatism... 1990)

Sur les partis politiques, à vrai dire, pas grand' chose. J'ai signalé le travail de Jacob Landau de 1957. Marius Deeb a bien rappelé en 1979 l'histoire parlementaire des années 1919-1939. (Party politics in Egypt : the Wafd and its rivals, 1919-1939). Et il y avait bien eu un article dans World Politics dans les années 1960 (dont je n'ai pas pu retrouver la trace) et l'on ne peut ignorer l'essai de Leonard Binder de 1966 (« Political recruitment and participation in Egypt », in M. Weiner et J. La Palombara, Political parties and political development). Aucune étude en tout cas, à ma connaissance, sur le Rassemblement de la Libération (1953) devenu l'Union nationale (1956) et son successeur l'Union socialiste arabe (1962), le régime qu'ils incarnent, les rôles qu'ils assument, le type de gouvernement qu'ils impliquent, la relation à la société qu'ils imposent. Pas de recherche conséquente non plus sur le Parti national démocrate et sur la réouverture partisane, légalisant quatre partis supplémentaires et permettant d'institutionnaliser le multipartisme, mais non le retour à un régime de type libéral parlementaire. La discipline est quasi muette sur la participation politique, sur les élections, le sens et la valeur de systèmes électoraux de type concurrentiel, sur leurs effets au niveau des acteurs, sur les nouvelles relations contradictoires entre ouverture politique interne et crise économique (et financière) externe impliquant une tutelle grandissante des organismes internationaux.

À tout prendre, le seul essai que nous possédions est un article d'Ilya Harik daté de 1974 (publié dans World Politics), réflexion sur les limites du parti unique. Puisqu'on mentionne à nouveau $\mathrm{ll} \mathrm{y}$ a Harik, il faut bien dire qu'il est aussi un des rares Américains de terrain, de l'Égypte intérieure et non seulement du Caire et de ses alentours. Son Political mobilization of peasants : a study of an Egyptian community paru en 1974 est le fruit d'une recherche d'ethnologie politique en milieu rural réalisée auparavant, qui doit moins à l'application d'un cadre théorique préalable qu'à une étude empirique des réalités. Celles-ci relèvent des liaisons, des modes de communication, de la circulation de l'information, entre villes et campagnes, dont l'auteur constate qu'elles existent et fonctionnent bel et bien dans le cas dont il s'occupe. Il faudra attendre 16 ans pour que Nathan Brown renoue avec le sujet les rapports entre paysannerie et État, mais non avec les techniques d'enquête ni l'investissement de terrain de son aîné (cf. Peasant politics in modern Egypt. The struggle against the State).

Il reste à dire un mot de réorientations récentes. Notamment d'ouvrages tendant à ne plus aborder l'Égypte par secteurs, mais de repartir en direction d'analyses en termes d'évolution, de transformation. Celles-ci ont permis un nouveau déplacement des focales depuis une dizaine d'années, avec l'économie en tête. En renversant la tendance définie en 1966 par le Britannique Patrick O'Brien qui avait étudié le passage de la libre entreprise au socialisme. Désormais, on observe le retour à la libre entreprise après l'échec du capitalisme d'État tel que décrit par Mark Cooper par exemple (IJMES, 1979), et les limites de l'interventionnisme étatique en matière agraire qu'a étudiées Simon Commander (en 1987), un autre britannique (il est vrai qu'avec Patrick O'Brien, Robert Mabro et Samir Radwan, l'Angleterre a possédé une équipe de spécialistes de l'économie égyptienne sans équivalent aux USA). changements de politiques et de structures, abordée par John Waterbury (lui-même 
influencé par l'ouvrage de Warren F. Ilchman et Norman T. Uphoff (The political economy of change, 1969) dans un maitre livre sans prétention théorique mais un des plus riches en informations et explications jamais écrit sur l'Égypte politique (The Egypt of Nasser and Sadat) qui date de $1983^{\circ}$. Alors que la plupart des politologues avaient jusqu'alors abordé les sujets d'ordre économique à titre secondaire, comme une sorte de contexte auquel on se devait de faire référence une bonne foi pour toutes, John Waterbury les considérait soudain de front, en eux-mêmes, les intégrant directement à l'explication politique, leur rendant leur valeur propre au sein du système interactif général. Deux ouvrages peuvent être considérés comme en étant les indispensables compléments, celui de Raymond Baker (Egypt's uncertain revolution under Nasser and Sadat)qui lui est antérieur (1978) et celui de Mark Cooper (The transformation of Egypt)de la même époque (1982).

Ce qui est proprement neuf, c'est que la génération qui arrive aux commandes intellectuelles et commence à occuper les postes académiques s'est attachée à des objets sans doute plus divers encore que celle qui l'a précédée. Mais, contrairement à celle-ci qui, à de rares exceptions près - P.J. Vatikiotis peut-être à propos de l'Armée, ll y a Harik sûrement quant aux relais entre centre urbain (du pouvoir) et périphérie rurale, et plus encore Leonard Binder se servant de son expérience égyptienne pour traiter de questions plus vastes, sans oublier Manfred Halpern dont le concept de nouvelle classe moyenne développé autrefois vient tout de même de l'observation des cas égyptiens - n'a pas tenté d'user d'un système politique pour construire des hypothèses valables pour d'autres, elle désire manifestement se servir du cas égyptien pour laisser son empreinte à un niveau de construction théorique plus général. Je renvoie à divers auteurs. À Ellis Goldberg, à partir des syndicats dont son expérience personnelle lui a permis de mieux connaître les modes d'organisation et les formes d'activité, (Tinker, tailor and textile worker... 1986). À Timothy Mitchell (Colonizing Egypt, 1988), appliquant les analyses d'un Derrida et d'un Foucault à la période anténassérienne, loin de la tradition académique made in US - et dont un très récent article d'ordre théorique sur les limites de l'État vient d'être publié par la très orthodoxe American Political Science Review ${ }^{10}$. À Robert Vitalis dont la thèse sur Aboud Pacha traite surtout d'une classe d'entrepreneurs issue de la bourgeoisie indigène. Ce qui ne se situe pas exactement dans l'axe des recherches antérieures d'inspiration néo-marxistes d'un Eric Davis (Challenging colonialism..., 1983), ou d'un Peter Gran (Islamic roots of capitalism : Egypt 1760-1840, 1979) mais en prolonge néanmoins les conclusions. À Robert Springborg aussi, mais à celui de Family, power and politics..., 1982, plutôt qu'à l'auteur du décevant Mubarak's Egypt, 1989. À Raymond Baker précité, dont diverses contributions publiées dix années après son Egypt's uncertain revolution..., 1978, démontrent qu'il vise lui aussi à transcender la spécificité égyptienne (cf. Sadat and after... 1990). Aux nombreux auteurs d'articles et de chapitres d'ouvrages collectifs portant sur les « résurgences » de la foi, la politisation du champ religieux et/ou l'islamisation du champ politique, les islamismes, reprenant et élargissant les travaux de Richard P. Mitchell de la fin des années 1960 sur les frères musulmans (The society of the muslim brothers, 1969).

Ramassons ce trop plein en quelques propositions.

711 - Recherches françaises et recherches américaines sont parties, en 1950, de positions inégalitaires vis-à-vis de l'Égypte, mais proches, ne serait-ce que parce que la discipline ne se structure vraiment et ne s'autonomise dans les deux pays qu'à partir de ce moment-là. Elles ont suivi ensuite des voies non parallèles. 
72 La France possédait une tradition académique, un capital culturel orientaliste, sans doute plus grands qu'aux USA - mais qui se situaient dans d'autres disciplines, en langue, en islamologie, en histoire -, sur lesquels les politologues n'étaient pas préparés à s'appuyer. Ils étaient, par ailleurs, trop prisonniers des conceptions et méthodes du droit public, plus formés à traiter des textes et organigrammes institutionnels théoriques que des sociétés en mouvement. Par intérêt académique aussi, la recherche sur le contemporain continuait à s'attarder sur le Maghreb ou le Croissant fertile plutôt que sur l'Égypte, pourtant considérée, depuis Mohamed Ali, comme l'intercesseur naturel de la relation entre l'Occident et le monde arabe. Ce qui explique sans doute pourquoi, à de très rares exceptions près (Pierre Mirel, Rémy Leveau) peu de chercheurs ont effectué des séjours au Caire, jusqu'au début des années 1980 en tout cas. Depuis lors, une nouvelle génération est venue se former sur place.

73 La recherche américaine, moins pourvue au départ du point de vue linguistique et islamologique, possédait, elle, un potentiel plus grand et n'était bridée par aucun complexe colonial. Elle a produit, en quantité et en qualité, $\mathrm{x}$ fois plus que son homologue française. À l'intérêt stratégique attaché à un pays considéré comme essentiel s'est ajouté l'intérêt académique de ceux qui se trouvaient sur place. Les supports institutionnels et matériels nécessaires à leurs recherches et, au retour aux États-Unis, la rémunération de leur thèse sur le marché du travail n'ont pas fait défaut. Et c'est un peu grâce aux études menées en Égypte que les politologues américains spécialistes du Moyen-Orient contemporain, en démontrant le caractère exceptionnel et spécifique de leur région devenue «domaine », se sont démarqués de l'ensemble de la communauté politologique - qui leur en tient rigueur -, même s'ils n'ont jamais constitué un groupe de pression aussi puissant que les africanistes par exemple. Enfin, les efforts récents pour sortir du cadre descriptif, pour alimenter la réflexion théorique à partir d'études réalisées dans la Vallée du Nil pourraient bien conférer à l'Égypte une place qui n'avait jamais été tout à fait la sienne à ce jour.

2 - C'est le mouvement déclenché par l'Armée, le 23 juillet 1952, qui a mobilisé du côté de la politologie ; celle-ci est restée après cela longtemps maîtresse du terrain, tout en changeant d'objectif au cours du temps. Le premier paradigme dominant, au cours des années 1960, fut celui d'un néo-despotisme oriental. État autoritaire, système non participant, structure hiérarchisée avec une armée et une bureaucratie aux commandes, une élite dirigeante quasi-cooptée, un leader charismatique, tout y était pour ne voir que les appareils d'État et ceux qui les contrôlaient. Seconde perspective, couvrant en gros la décennie 1970, mais elle débute avant et s'étend au-delà, celle d'une économie et d'une sociologie politiques prises au sens large et marquées, l'une et l'autre, par une préoccupation explicite pour les changements, sociaux et politiques, en correspondance ou interaction les uns avec les autres. Preuve d'une descente d'un cran dans l'échelle des objets et d'un retrait par rapport à l'étatique jusque-là prédominant. Preuve aussi de nouveaux regards vers ce que l'on nomme, abusivement et faute de mieux, la société civile. Nouvelle étape : celle, plus récente, de la recherche du politique au cœur de processus informels. Jusque-là peu observés : celle des élites locales, des groupes d'intérêt, des différentes activités, des comportements et expressions, des formes communautaires et associatives.

Nous voici presque à l'heure de la micropolitologie, celle des leaderships villageois, des communautés, des groupes. Elle risque d'appartenir à ceux qui auront les capacités de se rendre sur place, d'y effectuer des séjours de longue durée, non seulement d'en 
posséder la langue mais d'en connaître intimement la culture. À condition qu'ils n'oublient pas de se référer constamment aux avancées théoriques et méthodologiques concomitantes. Il restera alors aux visiteurs, aux chercheurs étrangers en transit, à collecter les produits des recherches effectuées sur place, à les emmener à New-York, Paris ou Londres, à les faire traiter par ordinateur interposé, en tirer les conclusions qui s'imposent et à les publier dans quelque revue académique; ce qui ajoutera à leur gloire ou aidera à leur promotion, mais peut-être pas vraiment à la connaissance de l'Égypte.

3 - L'Égypte, loin du centre jusqu'en 1952, aurait dû devenir centrale à partir de 1956, et centrale à différents titres, en raison des expériences politiques réalisées en son sein, mais aussi de sa position et de son influence aux niveaux du Tiers monde, du nationalisme arabe. Aussi pour des raisons stratégiques, parce qu'elle posait une équation politique de taille à l'Occident. Elle aurait dû rester centrale du point de vue de la recherche académique, pour comprendre les questions propres au développement économique, aux idéologies nationales, au marché politique. Passage obligé pour approcher sinon comprendre le Moyen-Orient, elle n'a jamais acquis, en fait, cette position prédominante. Hier encore, elle représentait un des points focaux des analyses politologiques essentiellement nord-américaines, au même titre que le Brésil, le Nigéria, l'Indonésie. Elle servait de terrain d'application, à partir duquel vérifier des hypothèses forgées dans les Universités de New-York, Boston, Washington, du Texas, de Californie, voire d'Europe... Aujourd'hui, l'Égypte a peut-être même perdu cette fonction-là. Elle a gagné, en échange, la possibilité de se retourner sur elle-même et de déterminer des hypothèses qui soient propres à son histoire et sa culture politiques. Car ce sont maintenant les Égyptiens qui mènent le train des recherches, pas tant ceux de l'Université américaine, que ceux du Centre d'études politiques et stratégiques d'AlAhram, du Centre National de la recherche criminologique et sociale et, plus encore, du Département de science politique et du Centre de Recherches et d'Etudes politiques de la Faculté d'Economie et de Science politique de l'Université du Caire.

\section{BIBLIOGRAPHIE}

\section{0}

- HEYWORTH-DUNNE (James), Religious and political trends in modern Egypt. Washington, Near and Middle East Monographs.

1951

- COLOMBE (Marcel), L'évolution de l'Égypte 1924-1950. Paris, Maisonneuve. 
1952

- BERTHIER (François), «L'idéologie politique des Frères Musulmans », Les Temps Modernes 83, septembre, pp. 541-556.

1954

- LANDAU (Jacob M.), Parliaments and parties in Egypt 1866-1953. New-York, Praeger. Version antérieure : Parliaments and parties in Egypt 1866-1934. Tel-Aviv, Israel Publishing Office (for the Israel Oriental Society). À l'origine, thèse de doctorat, soutenue à l'Université de Londres et portant sur la période 1866-1924.

\section{5}

- AMER (Adel), Les causes de la faillite du système constitutionnel égyptien 1923-1952. Paris, Thèse de droit public, Faculté de droit et d'économie.

1956

- LACOUTURE (Jean), LACOUTURE (Simonne), L'Égypte en mouvement. Coll. Esprit « Frontières ouvertes », Paris, Éditions du Seuil. (Nouv. éd. revue et augmentée, 1962).

- SPARROW (Gerald), The Sphinx awakes. London, R. Hale.

\section{7}

- BERGER (Morroe), Bureaucracy and society in modern Egypt. A study of the higher civil service. Princeton, Princeton University Press.

- LENGYEL (Emil), Egypt's role in world affairs. Washington, Public Affairs Press.

\section{8}

- ADAMS (Michael), Suez and after. Years of crisis. Boston, Beacon Press.

- BERTHIER (François), «L'idéologie sociale de la révolution égyptienne », Orient-6, pp. 49-72.

- KEDDOURIE (Elie), « Revolutionary Justice in Egypt : The Trial of 1953 », Political Quarterly, octobre-décembre, pp. 389-396.

- LANDES (David S.), Bankers and pashas : International finance and economic imperialism in Egypt. Cambridge, Mass., Harvard University Press.

- LITTLE (Tom), Egypt. Nations of the Modern World. London, Ernest Benn Limited.

- MIQUEL (André), « Patriotisme égyptien et nationalisme arabe », Orients, pp. 91-112.

1959

- WYNN (Wilton), Nasser of Egypt. Cambridge, Mass., Arlington Books. 
- AHMED (J.), The intellectual origins of Egyptian nationalism. London, Oxford University Press.

- AUSTRUY (Jacques), Structure économique et civilisation : l'Égypte et le destin économique de l'Islam. Paris, thèse de science économique, Faculté de droit et d'économie.

- MAKARIUS (Raoul), La jeunesse intellectuelle d'Égypte au lendemain de la seconde guerre mondiale. La Haye, Mouton.

\section{1}

- GARDNER (George Henry), Some social correlates of the transitional phase of change from the traditional to the modern way of life; an exploration among Egyptian secondary school and college youth, 1954-1955. Thèse (Ph. D), Princeton University.

- SAFRAN (Nadar), Egypt in search of political community ; an analysis of intellectual and political evolution of Egypt 1804-1952. Cambridge, Harvard University Press.

- VATIKIOTIS (Panayotis J.), The Egyptian army in politics : pattern for new nations? Bloomington, Indiana University Press (Nouvelle édition, Westport, Conn, Greenwood Press, 1975).

\section{2}

- ABDEL-MALEK (Anouar), Égypte : société militaire. Paris, Editions du Seuil. Traduction anglaise, Egypt : military society, New-York, Vintage Books, 1968.

- ABUL FATAH (Ahmad), L'Affaire Nasser. Paris, Plon.

- DELESTRE (E.), « Remarques sur le projet de la Charte nationale de la RAU », Orient-22, 3 ème trimestre.

\section{3}

- WAKIN (E.), A lonely minority : The modern story of Egypt's copts. New-York, Morrow.

\section{4}

- BINDER (Leonard), « Ideological foundations of Egyptian-arab nationalism » in Apter (David) ed. Ideology and discontent. New-York, Free Press of Glencoe.

- HARRIS (Christina P.), Nationalism and revolution in Egypt : the role of the muslim brotherhood. Stanford, The Hoover Institution on War, Revolution and Peace publié par Mouton, La Haye. Republié en 1981 par Hyperion Press, Westport, Connecticut.

- RIAD (Hassan), L'Égypte nassérienne. Paris, les Editions de Minuit.

- ZIEGLER (Jean), Sociologie de la nouvelle Afrique. Paris, NRF, « Idées ». (Cf. 4e partie, portant sur l'Égypte). 
1965

- ESTIER (Claude), L'Égypte en révolution. Paris, Julliard.

- MANSFIELD (Peter), Nasser's Egypt. London, Penguin African Library, (Edition revue et augmentée, 1969).

\section{6}

- O'BRIAN (Patrick), The revolution in Egypt's economic system : from private enterprise to socialism 1952-1966. London, Oxford University Press.

\section{7}

- BERQUE (Jacques), L'Égypte, impérialisme et révolution. Paris, Gallimard. Traduction anglaise 1972, Egypt : imperialism and revolution, (trad, par Jean STEWART), London, Faber and Faber. Du même auteur, voir Histoire sociale d'un village égyptien au XX'o siècle. La Haye, Mouton.

- CRECELUS (Daniel Neil), The Ulama and the State in modern Egypt. Thèse (Ph.D.) Princeton University. Voir aussi, du même auteur, «Al-Azhar in the Revolution », Middle East Journal, Winter 1966, pp. 31-49.

- L'Égypte révolutionnaire et socialiste. Paris, Éditions Le Communiste. (75 p. dont extraits de textes de Gamal Abdel Nasser, pp. 21-66).

- POWELL (Ivor), Disillusion by the Nile : what Nasser has done to Egypt. London, Solstice Productions.

- SAAD (Gabriel), The Egyptian agrarian reform 1952-1962. London, Oxford University Press.

\section{8}

- FLORY (Maurice), Mantran (Robert), Les régimes politiques des pays arabes. Paris, Presse universitaire de France. Collection «Thémis » (cf. pp. 89-96, 251-266).

- HOLT (P.M.) éd., Political and social change in modern Egypt. Oxford, Oxford University Press.

- SABER (Ali), Nasser en procès face à la nation arabe. Paris, Nouvelles Editions latines.

- SAREL (Sterngerg Benno), « Révolution par le haut dans les campagnes égyptiennes ». Les Temps Modernes, octobre.

\section{9}

- ABDEL-MALEK (Anouar), Idéologie et renaissance nationale. L'Égypte moderne. Paris, Anthropos.

- BAER (Gabriel), Studies in the social history of modern Egypt. Chicago, Chicago University Press.

- HANNA (Sami A), Gardner (George H), Arab socialism. A documentary Survey, with contributions by Sherif Mardin and Fayez Sayegh and a foreword by Aziz S. Atiya, Leiden, Brill.

- HUSSEIN (Mahmoud), L'Égypte : lutte de classes et libération nationale 1945-1967.Paris, Maspéro, Petite collection, 2 vol. ( $2^{\text {ème }}$ ed. 1945-1973, 1975). Voir aussi : La lutte des classes en Égypte de 1945 à 1968. Paris, Maspéro, 1969. $2^{\text {ème }}$ édition mise à jour (1945-1970), Paris, Maspéro, Cahiers libres 158-159, 1971. Traduction anglaise : Class conflict in Egypt, 1945-1970 de Michel Chirman et.al. NewYork,Monthly Review Press, 1973. 
- LACOUTURE (Jean), Quatre hommes et leurs peuples. Surpouvoir et sous-développement. Paris, Editions du Seuil.

- MilTCHELL (Richard P.), The society of the muslim brothers. London, Oxford University Press.

1970

-BE'ERI (Eliezer), Army officers in arab politics and society. London, Praeger-Pall Mall.

- BERGER (Morroe), Islam in Egypt today : social and political aspects of popularreligion. Cambridge, Mass., Cambridge University Press.

- MERRIAM (John G.), A study of the political modernization process in the liberal constitutional period Egypt 1918-1928. Ph. D. Indiana University (AAUMI).

\section{1}

- DEKMEJIAN (R. Hrair), Egypt under Nasir : a study in political dynamics. London, University of London Press. Albany, State University of New-York Press.

- LACOUTURE (Jean), Nasser. Paris, Editions du Seuil. Traduction anglaise Nasser, a political biography, par Daniel Hofstadter, New-York, Knopf, 1973.

- MAYFIELD (James B.), Rural politics in Nasser's Egypt. Austin, University of Texas Press. Voir aussi, du même auteur, Local institutions and Egyptian rural development. Ithaca, Rural Development Committee, Cornell University, 1974.

- STEPHENS (Robert), Nasser : a political biography. London, Allen Lane.

\section{2}

- BARBOUR (K.M.), The growth, location and structure of industry in Egypt. New-York, Praeger.

- EL-ASHRAM (Mohamed Abdel Halim), Nasser's ideology and organization : A modernizing experiment in Egypt 1952-1970. Ph. D., New-York University, (AAMUMI).

- FAKHOURI (Hani), Kafr el-Elow : an Egyptian village in transition. New-York,Holt, Rinehart and Winston.

- HEYKAL (Mohamed H.), Les documents du Caire. Traduit de l'anglais par H. Parisot, Paris, Flammarion.

- MAHFOUZ (Afaf EL-KOCHERI), Socialisme et pouvoir en Égypte. Préface de Maurice Duverger, Avant-propos de Boutros Boutros-Ghali, Paris, LGDJ, Pichon et Durand-Auzias.

- NUTTING (Anthony), Nasser. London, Constable.

\section{3}

- HAMID (Mohammed Bashir), Aspects of Nasir's ideological Revolution : The concepts ofarab socialism and arab unity (1952-1963). Ph. D. University of Sussex.

- WISSA-WASSEF (Cérès), « Le prolétariat et le sous-prolétariat industriel et agricole dans la RAU : formation, évolution, rôle politique ", in Kuray (Mubeccel B.) éd., Social stratification and development in the mediterranean basin. The Hague, Mouton. Voir aussi du même auteur, et de la même année « Le pouvoir et les étudiants en Égypte », Maghreb-Machrek 57, pp. 65-71. 


\section{4}

- HARIK (llya), The political mobilization of peasants : a study of an Egyptian community. Bloomington, Indiana University Press.

- HARIK (llya), « The single party as a subordinate movement », World Politics 26, 1973-74, pp. 80-105.

- PERLMUTTER (Amos), Egypt. The praetorian State. New Brunswick, N.J., Transaction Books.

- RADWAN (Samir), Capital formation in Egyptian industry and agriculture 1882-1967. St Antony's Middle East Monographs $n^{\circ}$ 2, London, Ithaca Press.

- REJWAN (Nissim), Hasserist ideology : its exponents and critics. New-York, Wiley (Shiloah Center for Middle Eastern and African Studies. The monograph series).

- TOMICHE (F.J.), Syndicalisme et certains aspects du travail en RAU (Égypte) 1900-1967. Paris, Maisonneuve et Larose.

\section{5}

- ABDEL FADIL (Mahmoud), Development, income distribution and social change in rural Egypt, 1952-1970. University of Cambridge, Department of Applied Economics occasional papers $n^{\circ} 45$, Cambridge, Cambridge University Press.

- AKHAVI (Shahrough), «Egypt : neo patrimonial elite », in Tachau (Frank) éd., Political elites and political development in the Middle East. New-York, Wiley.

- HANSEN (Bent), NASHASHIBI (Karim), 1975, Foreign trade regimes and economic development : Egypt. Special Conference Servies on Foreign Trade Regimes and Economic Development, National Bureau of Economic Research, vol. 4, New-York, Columbia University Press.

- HEYKAL (Mohamed H.), The road to Ramadan. New-York, Quadrangle, New-York Times Book, Co.

- JANKOSWSKIi (James P.), Egypt's young rebels. Stanford, Stanford University, Hoover Institution Press.

- SPRINGBORG (Robert), " Patterns of associations in the Egyptian political elite », in Political elites in the Middle East, (George Lenczowski éd.), Washington, American Enterprise Institute for Public Policy Research, pp. 83-108.

\section{6}

- BRADFORD (Gregory H.), The rise and fall of soviet influence in Egypt. Thèse, Naval Postgraduate School, Monterey, Calif.

- DAWISHA (Adeed I), Egypt in the arab world. The elements of foreign policy. London, Macmillan.

\section{7}

- BURELL (Robert Michael), Kelidar (Abbas R.), Egypt, the dilemnas of a nation 1970-1977. Berverly Hills, Calif. Sage Publications (The Washington Papers, 78 p.). L'Égypte d'aujourd'hui : permanence et changement 1805-1976. Paris, CNRS. [Chapitre d'Olivier Carré « Pouvoir et idéologie dans l'Égypte de Nasser et de Sadate ", pp. 243-266]. 
- ELSHEIKH (Ibrahim), Massmedia and ideological change in Egypt (1950-1973). Thèse, Amsterdam, Universiteit van Amsterdam.

- GELLNER (Ernest), Waterbury (John), ed., Patrons and clients in mediterranean societies. London, Duckworth.

- HARIK (llya), « Mobilization policy and political change in rural Egypt » in Harik (llya) and Antoun (Richard) ed., Rural politics and social change in the Middle East. Bloomington, Indiana University Press, 1972, pp. 287-314. Aussi in Hopkins (Nicholas S.), Ibrahim (Saad Eddin), Arab society in transition. Le Caire, The American University in Cairo Press, 1990.

- NARAYAN (B.K.), Anwar el-Sadat : man with a mission. New-Delhi, Vikas Publishing House.

- RUBINSTEIN (Alvin), Red star on the Nile. Princeton, Princeton University Press.

- SMITH (David B.), The Egyptian military elite. Thèse, Naval Postgraduate School, Monterey, Calif.

-SYKES (William G.), Egyptian arms procurement in the post 1973 war era : A case study in the dynamics of the arms diversification process. Thèse, Naval Postgraduate School, Monterey, Calif.

\section{8}

-BAKER (Raymond), Egypt's uncertain revolution under Nasser and Sadat. Cambridge, Harvard University Press.

-BINDER (Leonard), In a moment of enthusiasm. Political power and the second stratum in Egypt. Chicago, University of Chicago Press.

- CARRÉ (Olivier), « Utopies socialistes en terre arabe d'Orient », Revue Tiers-Monde, 75, JuilletSeptembre.

- DESSOUKI (Ali Eddine Hilal) ed., Democracy in Egypt : problems and prospects ? Cairo, American University in Cairo, Cairo Papers in Social Science Monographs.

-HEYKAL (Muhamed H.), Sphinx and Commissar : The rise and fall of soviet influence in the arab world. London, Collins, Traduction française Le Sphinx et le Commissaire : heurs et malheurs des Soviétiques au Moyen-Orient. Paris, Jeune Afrique, 1980.

- SPRINGBORG (Robert), « Professional syndicates in Egyptian politics, 1952-1970 ». International Journal of Middle East Studies 9, pp. 275-295.

-TRIMBERGER (Ellen Kay), Revolution from above : military bureaucrats and development in Japan, Turkey, Egypt and Peru. New Brunswick, Transaction.

- VATIKIOTIS (Panayotis J.), Nasser and his generation. London, Broom Helm.

-WATERBURY (John), Egypt, burdens of the past, options for the future. Bloomington, Indiana University Press. (A l'origine : Hanover, N.H., The American Universities Field Staff).

1979

- CARRÉ (Olivier), La légitimation islamique des socialismes arabes : analyse conceptuelle combinatoire de manuels scolaires égyptiens, syriens et irakiens. Paris, Presses de la Fondation Nationale de Science Politique.

- COOPER (Mark), « Egyptian state capitalism in crisis : economic policies and political interests, 1967-1971 ». International Journal of Middle East Studies 10, pp. 481-516. 
- DEEB (Marius), Party politics in Egypt : The Wafd and its rivals 1919-1939. London, Ithaca Press. (Middle East Centre, St Antony's College, Oxford).

- HARIK (llya), Socio-economic profile of rural Egypt. Le Caire, International Center for Population Studies and Research, (Rapport),

- NASSERr (Munir K.), Press, politics and power : Egypt's Heikal and al-Ahram, Ames, Iowa State University Press.

- SHOUKRI (Ghali), Égypte. La contre-révolution. Préface de Jacques Berque, Paris, Le Sycomore, Version anglaise, Egypt : portrait of a president 1971-1981. The counter-revolution in Egypt. Sadat's road to Jerusalem. (Préface supplémentaire de l'auteur). London, Zed Press, 1981.

- WATERBURY (John), Hydropolitics of the Nile valley. Syracuse, Syracuse University Press.

\section{0}

- ABDEL FADIL (Mahmoud), The political economy of Nasserism. Cambridge,Cambridge University Press.

- ANTHONI (David J.), Political culture and the nature of political participation in Egypt. Thèse Naval Postgraduate School, Monterey, Calif.

- AYUBI (Nazih N.M.), Bureaucracy and politics in contemporary Egypt. London, Ithaca Press. (Middle East Center, St Antony's College, Oxford).

- MEYER (Gail), Egypt and the United States : the formative years. Rutherford, N.J., Fairleigh Dickinson University Press.

- MOORE (Clement Henry), Images of development. Egyptian engineers in search of industry. Cambridge, Mass., London The Massachussets Institute of Technology Press.

- SAYED (Salah), Cases in management (Egypt and Sudan). Cairo, Graduate Management program. American University in Cairo.

- SHAZLY (Saad), The crossing of the Suez. San Francisco, American Mideast Research.

- VATIKIOTIS (Panayotis, J.), The History of Egypt from Muhamad Ali to Sadat. $2^{\circ}$ éd., London, Weidenfeld, Baltimore, The Johns Hopkins University Press.

1981

- EFRAT (Moshe), The defence burden in Egypt during the deepening of the Soviet involvement in 1962-1973. Ph. D. thesis, University of London.

- FARID (Adel Majid), Stephens (Robert), Auda (Muhammad), Nasser, a reassessment. London, Arab Research Centre.

- GERSHONI (Israel), The emergence of pan-arabism in Egypt. Shiloah Center for Middle Eastern and African Studies, Tel-Aviv University.

- HIST (David), Beeson (Irene), Sadat. London, Faber and Faber.

- IKRAM (Khalid), Egypt : economic management in a period of transition. Baltimore, Johns Hopkins University Press.

- ISRAELI (Raphael), «I, Egypt » : aspects of president Anwar al-Sadat's political thought. Jerusalem, Magnes Press, Hebrew University. 
- RICHARDS (Allan), Egypt's agricultural development : technical and social change 1800-1980. Boulder, Co., Westview Press.

- HAFIK (Fouad Fahmy), The press and politics of modern Egypt 1798-1970. A comparative analysis of casual relationships. 2 vol. Ph. D. New-York University. (AAMUMI).

- SULLIVAN (Earl L.), KORAYEM (Karima), Women and work in the arab world. Cairo, American University in Cairo.

1982

- ABDEL-KHALEK (G.), Tignor (Robert) ed., The political economy of income distribution in Egypt. NewYork Holmes \& Meier.

- AYUBI (Shaheen), Foreign policy making in the arab republic of Egypt : the role of leadership in decision

- making 1970-1972. Ph. D. University of Pennsylvania. (AAMUMI).

- BALTA (Paul), Rulleau (Claudine), La vision nassérienne. Textes choisis et présentés par P.B. et C.R., Paris, Sindbad.

- COOPER (Mark N.), The transformation of Egypt. London, Croom Helm.

- FERNANDEZ-ARMESTRO (Felipe), Sadat and his statecraft. London, Kensal Press.

- FRIEDLANDER (Melvin A.), The management of peace-making in Egypt and Israël 1917-1979. Ph. D. The American University. (Ann Arbor, Michigan, University Microfilms International, 1982).

- IBRAHIM (Saad Eddin), « Egypt's islamic militants », MERIP Reports 103, pp. 5-14, reproduit in Hopkins (Nicholas S.), Ibrahim (S.E.), Arab society. Social science perspectives. Le Caire, The American University in Cairo Press, 1985, pp. 494-507.

- MEIRING (Desmond), Fire of Islam. London, Wildwood House.

- MIREL (Pierre), L'Égypte des ruptures : l'ère Sadate de Nasser à Moubarak. Paris, Sindbad.

- SPRINGBORG (Robert), Family, power and politics in Egypt. Sayed Bey Marei. His clan, clients and cohorts. Philadelphia, University of Pennsylvania Press.

- TERRY (Janice J.), The Wafd, 1919-1952 : corner stone of egyptian political power. London, Third World Centre for Research and Publishing.

- TILDON (Ralph Butler), Prelude to war : the Egyptian decisions of 1967 and 1973. Ph. D. Columbia University, New-York. (Ann Arbor, Michigan, University Microfilms International, 1982).

\section{3}

- DAVIS (Eric), Challenging colonialism. Bank Misr and Egyptian industrialization 1920-1941. Princeton, Princeton University Press.

- FAHIM (Husseim M.), Egyptian Nubians. Resettlement and years of coping. Salt Lake City, University of Utah Press.

- FRIEDLANDER (Mervin A.), Sadat and Begin : the domestic politics of peacemaking. Boulder, Co., Westview Press.

- HEYKAL (Mohamed H.), L'automne de la colère. L'assassinat de Sadate. Paris, Ramsay, Traduction anglaise Autumn of fury. Trad. allemande Das ende eines Paraoh : eine politische biographie. Dusseldorf, Becon, 1984. 
- WARBURG (Gabriel R.), KUPFERSCHMIDT (Uri M.), 1983, Islam, nationalism and radicalism in Egypt and the Sudan. New-York, Praeger Publications.

- WATERBURY (John), The Egypt of Nasser and Sadat. The political economy of two regimes. Princeton, Princeton University Press.

\section{4}

- AMER (M. Y.), Egypt between the superpowers : continuity of change in Egyptian foreign policy under Mubarak. Thèse Naval Postgraduate School, Monterey, Calif.

- BATATU (Hanna), The Egyptian, Syrian and Iraqi revolutions : some observations on their underlying causes and social character. Inaugural Lecture. The Shaykh al-Salem al-Sabah Chair in Contemporary Arab Studies, Washington DC. Center for Contemporary Arab Studies, Georgetown University, $29 \mathrm{p}$.

- CARRÉ (Olivier), Mystique et politique, lecture révolutionnaire du Coran par Sayyid Qotb, frère musulman radical, 1906-1966. Paris, Presses de la Fondation Nationale de Science Politique.

- KAYS (Doreen), Frogs and scorpions. Egypt, Sadat and the media. London, Frederick Muller Limited.

- KEPEL (Gilles), Le Prophète et Pharaon : les mouvements islamistes dans l'Égypte contemporaine. Préface de Bernard Lewis. Paris, La Découverte, Traduction anglaise Muslim extremism in Egypt. The Prophet and Pharaoh. Berkeley and Los Angeles, University of California Press, 1985.

- MATTHEWS (Roger), Economic and political trends in Egypt. Washington DC, Middle East Institute. - VATIKIOTIS (Panayotis J.), Arab and regional politics in the Middle East. New-York, St Martin Press, London, Croom Helm.

\section{5}

- ABDALLA (Ahmed), The student movement and national politics in Egypt, 1923-1973. London, Al-Saqi Books (distrib. Zed Books).

- BURNS (William J.), Economic aid an American policy toward Egypt 1955-1981. Albany, State University of New-York Press.

- HASSOU (Tawfiq Y.), The struggle for the Arab world: Egypt's Nasser and the Arab League. London, KPI.

- HINNESBUSCH (Raymond A.), Egyptian politics under Sadat. The post-populist development of an authoritarian - modernizing State. Cambridge, New-York, Cambridge University Press.

- HOPKINS (Nicholas S.), «The Political Economy of Two Arab Villages » [Testour en Tunisie, Musha en Égypte], in Hopkins (N.S.), Ibrahim (Saad Eddine), Arab Society. Social science perspectives. Le Caire, The American University in Cairo Press, pp. 307-321.

- HOPWOOD (Derek), Egypt, politics and society 1945-1984. 2ème ed., Boston, London, Sydney, Allen and Unwin.

- LOPEZ GARCIA (Bernabe), FERNANDEZ SUZOR (Cecilia), Introduccion a los regimenes y constituciones arabes. Madrid, Centra de estudios constitucionales. Voir « Primera Parte : proximo oriente. Capitulo I : Egipto, pp. 28-66.

- WARBURG (Gabriel R.), Egypt and the Sudan : studies in history and politics. London, Frank Cass. 
1986

- ANSARI (Hamied), Egypt, the stalled society. Albany, State Univ. of New-York Press.

- ARONSON (Geoffrey), From sideshow to center stage. US policy toward Egypt 1946-1956. Boulder, Lynne Rienner Publishers.

- CARTER (Barbara Lynn), The Copts in Egyptian politics 1918-1952. London, Dover, New-York, Croom Helm.

- FARAH (Nadia Ramsis), Religious strife in Egypt : crisis and ideological conflict in the seventies. NewYork, Gordon and Breach Science Publishers.

- GERSHONI (Israel), Jankowski (James P.), Egypt, islam and the Arabs : the search for Egyptian nationhood 1900-1930. New-York, Oxford University Press.

- GOLDBERG (Ellis), Tinker, tailor and textile worker. Class and politics in Egypt 1930-1952. Berkeley, Los Angeles, London, University of California Press.

- GUENENA (Nemat), The " Jihad » : an « Islamic alternative » in Egypt. Cairo, American University in Cairo Press.

- JANSEN (Johanes J.G.), The neglected duty : the creed of Sadat's assassins and islamic resurgence in the Middle East. New-York, McMillan.

- KRÄMER (Gudrun), Ägypten unter Mubarak : identität und nationales interesse. Baden-Baden, Nomos.

- SULLIVAN (Earl L.), Women in Egyptian public life. Syracuse, N.Y., Syracuse University Press.

- WEINBAUM (Marvin G.), Egypt and the politics of US economic aid. Boulder, London, Westview Press.

\section{7}

- BEININ (Joël), LOCKMAN (Zachary), Workers on the Nile : nationalism, communism, Islam and the Egyptian working class, 1882-1954. Princeton, Princeton University Press.

- COMMANDER (Simon), The State and agricultural development in Egypt since 1973. London, The Ithaca Press.

- HEYKAL (Mohamed H.), L'affaire de Suez : un regard égyptien. Traduit de l'anglais par C. Yelnick, Paris, Ramsay.

\section{8}

- BOTMAN (Selma), The rise of Egyptian communism 1939-1970. Syracuse, Syracuse University Press. - GOLDSCMIDT (Arthur Jr.), Modern Egypt : the formation of a nation state. Boulder, Co. University Press.

- HARRIS (Lilian Craig) ed., Egypt, internal challenges and regional stability. London, New-York, Routledge and Kegan Paul.

- MCDERMOTT (Anthony), Egypt from Nasser to Mubarak : a flawed revolution. London, New-York, Croom Helm.

- MITCHELL (Timothy), Colonising Egypt. Cambridge, UK, Cambridge University Press. 
- PALMER (Monte), LEILA (Ali), YASSIN (El-Sayed), The Egyptian bureaucracy. Syracuse, Syracuse University Press.

1989

- KITROEFF (Alexander), The Greeks in Egypt, 1919-1937 : Ethnicity and class. Oxford, St Antony's Middle East Monographs, $\mathrm{n}^{\circ} 20$.

- KRÄMER (Gudrun), The Jews in modern Egypt 1914-1952. Seattle, The University of Washington Press.

- LIPPMAN (Thomas W.), Egypt after Nasser. Sadat, peace and the mirage of prosperity. New-York, Paragon House.

- SPRINGBORG (Robert), Mubarak's Egypt, fragmentation of the political order. Boulder, Westview Press.

- TRIPP (Charles), Owen (Roger) ed., Egypt under Mubarak. London, New-York, Routledge.

- ZAALOUK (Malak), Power, class and foreign capital in Egypt. The rise of the new bourgeoisie. London and New Jersey, Zed Books.

1990

- BAKER (Raymond), Sadat and after : strugglers for Egypt political soul. Cambridge, Mass., Harvard University Press.

- BIANCHI (Robert), Unruly corporatism : associational life in twentieth-century Egypt. New-York, Oxford University Press.

- BROWN (Nathan J.), Peasant politics in modern Egypt. The struggle against the State. New Haven and London, Yale University Press.

- KORANY (Bahgat), chapitre sur l'Égypte in Les régimes politiques arabes. 2ème éd., Paris, Presses Universitaires de France, pp. 203-287.

- OWEISS (Ibrahim M.) Dir, The political economy of centemporary Egypt. Washington DC, Center for contemporary Arab Studies, Georgetown University.

- REEVES (Edward B.), The hidden government, ritual, clientelism, and legitimation in northern Egypt. Salt Lake City, University of Utah Press.

- RICHARDS (Allan), Waterbury (John), A political economy of the Middle East : State, class and economic development. Boulder, Co., Westview Press.

- RUBIN (Barry M.), Islamic fundamentalism in Egyptian politics. New-York, St Martin Press.

- TOLEDANO (Ehud R.), State and society in mid-nineteenth century Egypt. Cambridge, New-York, Cambridge University Press.

\section{Quelques exemples d'autres études en langues étrangères}

1967

- DADIANI (Lionel lakovlevich), Gosurdarstvenyi stroi Ob edinennoi Arabskoi Respubliki. Moskva, Ordi. 
1969

-SIDOR (Kazimierz), Naseryzm : historia, pratika i teoria socjalizmu w ZRA. Warszawa, Wiedza Powzechna.

\section{2}

- BUREN (Rainer), Nassers Àgypten als arabisches Verfassungs Modell. Opladen, Leske.

\section{7}

- FRESCOBALDI (Dino), La sfida di Sadat. Milano, Rizzoli.

- HANSEN (Gerda), Wirtschaft, Gesellschaft und Politik Agyptens : e. Bibliogr. Einf. Hamburg, Orient Institut. (Introduction bibliographique).

\section{8}

- ERLIKH (Hagai), Ha-Studentim be-mishtar Sadat, 1970-1977. Tel-Aviv, Mekhon Shiloah le-heker haMizrah ha-tikhon ve-Afrikah, Universitat Tel-Aviv.

\section{9}

- GAMMER (Mosheh), Igude poalim be-Mitsrayim bi-tekufat Sadat. Tel-Aviv, Mekhon Shiloah leheker ha-Mizrah ha-tikhon ve-Afrikah, Universitat Tel-Aviv.

1980

- GOMAA (Farouk), Ägypten während der ersten Zwischenzeit. Wiesbaden, Reichert.

\section{4}

- KOSHELEV (Vladimir Sergeevich), Egipet, uroki istorii : borba protiv kolonialogo gospodstva $i$ kontrr evoliutsii 1879-1981. Minsk, Izd-vo « Universitetskoe ».

\section{6}

- KNIAZEV (Aleksandr Grigorevich), Egipet posle Nasera, 1970-1981. Moskva, Izd-vo « Nauka ».

\section{ANNEXES}

\section{Sources}

Du point de vue de la critique des disciplines, je renvoie à un ouvrage dirigé par Hisham Sharabi, Theory, politics and the Arab world, critical responses, 1990 et, plus spécialement, 
au chapitre rédigé par Liza Anderson : « Policy-making and theory building : American political science and the islamic Middle East », pp. 52-80. Voir aussi l'article d'Abdallah Saaf (Al-Ahsas $n^{\circ} 72$, pp. 13-17) sur « Nasser et le nassérisme sous le regard occidental ». Sur la science politique française, on retrouvera ici des éléments empruntés à Pierre Favre, Yves Schemed, François d'Arcy et al., extraits de " Sciences politiques ", chapitre de L'État des sciences sociales en France, Paris, Editions La Découverte, 1986, pp. 269-294, ainsi que d'autres nés de la lecture de textes signés Michel Camau, Jean Leca, Rémy Leveau ou de conversations avec ces mêmes auteurs.

Pour ce qui est du traitement réservé à l'Égypte par la science politique américaine, je me réfère à une production à tout prendre importante et dont la bibliographie donne une idée. J'ai aussi bénéficié des nombreuses et précieuses suggestions et remarques de : Ali Eddine Hilal Dessouki, Directeur du Centre d'études et de recherches politiques de la Faculté d'Economie et de Science politique de l'Université du Caire, Afaf Mahfouz, Professeur de science politique de l'Université d'Helouan au Caire, ll y a Harik, Directeur du Centre américain de recherche en Égypte, Tim Sullivan, Professeur de science politique à l'Université américaine du Caire (AUC), John Waterbury, Professeur de science politique à l'Université de Princeton. La comparaison avec l'anthropologie est le fruit d'un essai antérieur et des précieuses informations fournies par Nicholas Hopkins, Professeur à AUC, dont il sera fait état dans un autre texte. Que tous trouvent ici l'expression de ma profonde reconnaissance et acceptent mes excuses s'ils estiment que mes propos ne reproduisent pas fidèlement les leurs, a fortiori les trahissent.

\section{Éléments bibliographiques}

Les éléments d'une bibliographie sélective, présentés ici, rassemblent pour l'essentiel des ouvrages avec mention exceptionnelle de quelques thèses et articles, traitant de ce que l'on appellera l'Égypte politique, soit l'Égypte considérée du point de vue de la politologie. Toutefois, à côté de travaux relevant explicitement de la science politique, figurent des études historiques et économiques, des livres écrits par des journalistes, des témoignages... susceptibles d'aider à la saisie de l'univers politique. Sont répertoriés des écrits publics en français et en anglais, y compris de traductions de l'arabe.

À titre de simple illustration ont été ajoutés, in fine, quelques titres d'ouvrages parus dans d'autres langues que l'arabe. Les travaux en langues européennes font l'objet d'autres recensions.

Contrairement aux usages et pour appuyer l'exposé, la liste est établie selon l'ordre chronologique.

\section{NOTES}

1. Je dois préciser que j'emploierai indifféremment politologie, science politique, sociologie politique. Il va aussi de soi que les dénominations «science politique française " et "science politique américaine » n'interviennent ici que pour les besoins de la présentation. On sait qu'à l'intérieur de ces cadres artificiels existent des catégories fort différentes et que, par exemple, 
fonctionnalistes et néo-marxistes américains n'ont guère en commun, pas plus que constitutionnalistes et économicistes français, par exemple.

2. Si l'on peut introduire un élément personnel dans ce propos, je dirai que si je n'avais pas lu ce livre à Alger lors de sa publication, il ne me serait jamais venu à l'idée de rebasculer tout un enseignement sur les institutions politiques algériennes et de repartir en 1830, pour ne plus piétiner dans l'embrouillamini des événements dont j'étais le témoin, puis d'en faire la matière d'un livre.

3. Pensons aux formules de Michael Hudson dans Arab politics. The search for legitimacy (1977, cf. pp. 234-251), ouvrage de référence, quasi manuel faudrait-il dire, pour tout étudiant suivant un cours sur le monde arabe contemporain. « To be sure, the Egyptian political system today faces a serious legitimacy problem, but as a society and political culture, Egypt, by virtue of its differenciated and elaborate structures and value patterns, is the most advanced in the Arab world. Accordingly, its experience in developing a revolutionary legitimacy is of interest not just because of Egypt's intrinsic weight in Arab affairs but also for insights into the future of other systems in the region» (pp. 234-235). "It (Egypt) is, of course, one of the most homogeneous societies in the Arab world» (p. 235). «Egypt began to develop a modern consciousness and modern structures from just after the middle of the nineteenth century, when most other Arab societies were under traditional local leaders» (p. 236). "Long the intellectual, cultural, and religious center of the Arab world, its leaders, especially after the Nasir revolution, saw a political role as well for Egypt throughout this area » (p. 240).

4. Différence de traitement d'avec la production française : je vais me limiter à la production académique, pour l'essentiel.

5. Cf. bibliographie.

6. Notons, toutefois, que si l'enseignement s'est poursuivi à l'université américaine du Caire et que des politologues purent y enseigner, les recherches ont été pratiquement interrompues pendant les dernières années de l'ère nassérienne, après la défaite de 1967.

7. Selon la démonstration apportée par Robert C. Tucker, lui-même historien de l'économie égyptienne contemporaine (Modernization and British colonial rule in Egypt, 1982-1914, 1966), cf. « The theory of charismatic leadership », Daedalus, Summer 1968, pp. 731-756.

8. Je me permets de renvoyer aux textes que j'ai rédigés en conclusion des Actes de colloques récents: Etudes politiques du monde arabe d'une part et Modernisation et nouvelles formes de mobilisation sociale Egypte-Brésil, Le Caire, CEDEJ, 1990 et 1991.

9. Lors des interviews et entretiens non directifs avec des chercheurs égyptiens anglophones et américains, à la question «Quel ouvrage recomanderiez-vous aux étudiants et chercheurs pour le mieux appréhender l'Egypte politique actuelle?» la majorité a répondu en citant The Egypt of Nasser and Sadat.

10. Mitchell, Timothy, «The limits of the State : beyond statist approaches », march 1991, vol 85 $\mathrm{n}^{\circ}$ 1, pp. 77-96.

\section{INDEX}

Mots-clés : politologie, science politique 
AUTEUR

JEAN-CLAUDE VATIN

CNRS/Cedej 